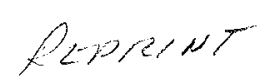

Department of Meteorology, University of Wisconsin-Madison, Madison, Wisconsin, U.S.A.

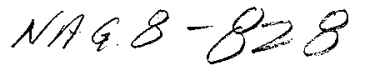

IN-4十:C

\title{
An Explicit Three-Dimensional Nonhydrostatic Numerical Simulation of a Tropical Cyclone
}

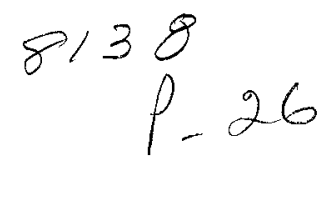

\section{G. J. Tripoli}

With 12 Figures

Received January 31, 1992

Revised June 28, 1992

\section{Summary}

A nonhydrostatic numerical simulation of a tropical cyclone is performed with explicit representation of cumulus on a meso- $\beta$ scale grid and for a brief period on a meso- $\gamma$ scale grid. Individual cumulus plumes are represented by a combination of explicit resolution and a 1.5 level closure predicting turbulent kinetic energy (TKE).

The results demonstrate a number of expected and unexpected important scale interaction processes. Within the central core of the developing cyclone, meso- $\beta$ convective regions grow and breakdown into propagating inertiagravity waves throughout the lifecycle of the cyclone. In the early stages, the amplitude of pressure fluctuations associated with the meso- $\beta$ scale convection exceed the central pressure of the cyclone and strongly modulate its intensity. With each meso- $\beta$ scale pulsation, the cyclone core increases in strength, measured by the central pressure deficit. The increasingly strong inertial frequency of the storm core acts to increasingly trap the convection induced heating within the core by balancing the tangential wind against the low central pressure, before the meso- $\beta$ scale convection breaks down and sends the warmth away as a propagating wave. Eventually, the slow manifold's amplitude exceeds the amplitude of the meso- $\beta$ scale oscillations and a stable eye region is formed. As inertial instability increases, increasingly high thermal warmth can be protected in the core, allowing persistent subsidence to form and to clear out the cyclone eye.

On the outside of the eye wall, strong inertial stability gradients in the troposphere cause convective warming to split the inflow to the eye wall and spawn outwardly propagating inertia gravity waves. These waves carry away all of the heating forced by convection that is not inertially trapped by the eye wall and act as a moderating influence on storm intensity.
Inertia gravity waves are also spawned in the stratosphere at the top of the eye wall by the revolution of asymmetric cumulus structures. In all instances, the tropospheric waves are coupled to the propagating stratospheric waves which both move at $35 \mathrm{~ms}^{-1}$, although there are many instances where the stratospheric waves seem to have no tropospheric counterpart. Hence the anvil top forcing and low level breakdown are linked.

The outwardly propagating inertia gravity waves act to initiate outer bands of convection. This initiation is with the assistance of low level boundary layer variations of density related to previous convection and to virga falling from the anvil which moistens and destabilizes the mid levels of $\theta_{e}$ minimum. The convection initiated by these waves does not move substantially outward with the wave, although may appear to develop outward discontinuously.

\section{Introduction}

The notion of CISK (Conditional Instability of the Second Kind) was first described by Charney and Elliasen (1964) as a way to explain the growth of a hurricane from a weak tropical depression. The underlying principle is that an ensemble of deep precipitating cumulus result in deep tropospheric heating which lowers the surface pressure within the storm, increasing the tangential winds of the balanced vortex. The friction layer induces radial inflow which, in turn, forces the convergence of warm moist marine layer flow into the storm to fuel the convection. The critical link in this chain of events was the relationship between 
the surface convergence and the heating function which is the role played by deep cumulus convection.

Early attempts to model this interaction by Ooyama (1964) were thwarted by the existence of nonlinear instability. Charney and Elliasen (1964) were successful in achieving vortex growth because they introduced the concept of cumulus parameterization which attempted to represent the effects of cumulus without explicitly resolving cumulus circulations. Their success together with Ooyama's experiences led to massive observational and parameterization development efforts in the community to accurately define the heating function hoping that tropical cyclone genesis could be accurately predicted. Parameterization theory spread to other areas of meteorological prediction and theoretical analysis where cumulus were deemed important, such as general circulation theory (Arakawa and Schubert, 1974), wave CISK theory (Lindzen, 1974; Raymond, 1975; Raymond, 1976), mesoscale convective systems (Fritsch and Chappel, 1980; Kreitzberg and Perky, 1976) and so on. In all applications, the use of cumulus parameterization schemes to represent scale interaction has resulted in little or no increase in the knowledge of the processes they were designed to represent. As model resolution increases to resolve the meso- $\beta$ scale, the underlying cumulus parameterization assumption of scale separation breaks down.

The development of three dimensional cumulus/mesoscale models in the mid 1970s (Klemp and Wilhelmson, 1978; Cotton and Tripoli, 1978; Clark, 1977) led to massive breakthroughs in the 1980 s in the understanding of cumulus scale overturning and gravity wave behavior. At the same time, scientists were beginning to recognize important aspects of the CISK process beyond the effects of Ekman pumping which were highly dependent upon the structures of the small circulations within the cumulus ensemble. For instance, gravity waves were proposed as a major component of spiral bandedness in tropical cyclones (Kurihara, 1976; Willoughby, 1977; Willoughby, 1978; Willoughby, 1979), the degree to which inertia gravity waves were spawned by deep convection was shown to strongly modulate the cyclone growth rate (Schubert and Hack, 1982), and the existence of asymmetric wave-like features in the cyclone circulation were shown to significantly affect radial momentum transport (Holland, 1983).
In order to build a scale interaction threedimensional model of a tropical cyclone, all thermodynamical and dynamical processes must be represented simultaneously. Hydrostatic, axisymmetric, and cumulus parameterized models are inadequate for this purpose because of the built-in biases and restrictions inherent in their design.

Since Rosenthal (1978) demonstrated that Ooyama's (1964) concern of nonlinear instability was somewhat overstated and that explicit convection models on the tropical cyclone scale were possible, there has been renewed interest in explicitly representing latent heat release rather than parameterizing it. With model resolutions allowed under present computing constraints in three dimensions, it is still not quite possible to realistically resolve the true scale of cumulus plumes. So the debate becomes one of whether the parameterized approach, with assumed subgrid scale cumulus ensembles with clouds of assumed simplistic structures, is more realistic or whether explicitly predicted convective overturning on the scale of mesoscale convective systems rather than individual plumes, is more realistic in a model of the entire cyclone.

In a relatively short while, that debate will become irrelevant because plausible model resolutions will dramatically increase so that cumulus can be explicitly resolved realistically. In this paper a nonhydrostatic tropical cyclone simulation will be presented which has sufficient grid resolution to explicitly resolve convective overturning on the full meso- $\beta$ scale, and for a shorter period of simulation, a portion of the meso- $\gamma$ scale near the eye wall. Unresolved convection was represented by a 1.5 level turbulence closure scheme.

The purpose of this paper is to explore the types of internal scale interactions which arise in a fully three-dimensional non-hydrostatic model of a tropical cyclone when cumulus are explicitly represented. As a consequence, it is desirable to keep the interaction with the initial state and the initial environment as simple as possible so the results can be easily understood. It is elected to simulate the growth of an axi-symmetric modified Rankine vortex perturbation in an initial undisturbed marine environment with thermodynamic structure typical for tropical cyclone development and without mean wind. 
The next section will describe the numerical model employed briefly and will be followed by a section discussing the experimental design. Next the results will be presented for a 56 hour simulation period. This will be followed by a section which attempts to tie the results together to form a coherent picture of the scale analysis process as it can be modeled today. Finally, in section 6 , conclusions concerning the implications of these results will be drawn.

\section{Numerical Model}

The numerical model used was the Tripoli (1992) nonhydrostatic mesoscale model. The model was based on the non-Boussinesq quasi-compressible dynamical equations. Model thermodynamics were calculated by integrating the enthalpy conserving $\theta_{i l}$ along with the total water and several ice and liquid water precipitating hydrometeor specific humidities. The thermodynamic system was closed under the assumption of zero supersaturation over liquid which also defined diagnostic relationships for vapor and cloud water. For these simulations, it was assumed that the entire grid lies over a body of water and so the model's terrain following coordinate system was not implemented. A spherical horizontal coordinate transformation was implemented for this simulation.

This numerical experiment utilized no cumulus parameterization despite its meso- $\beta$ scale horizontal resolution over the first 50 hours of simulation. In this way wave scale interactions generated by the convective motions could be simulated explicitly, although at times these motions could significantly depart from realism due to deficiencies of the resolution. Some of the limitations of this approach were softened by utilizing a 1.5 level turbulence closure, where turbulent kinetic energy (TKE) was predicted using a modified form of the closure developed by Redelsperger and Someria (1981, 1982), and subsequently used to form the vertical down-gradient mixing terms. Some modifications were included to represent the effects of saturation, ice phase, and precipitation loading on turbulence generation. A more complete explanation of the closure is given in Appendix A.

The 1.5 level closure enabled the mixing effects of cumulus to be transported by the explicitly resolved motions. Upscale transport, such as that caused by subgrid scale motions transporting subgrid scale fluctuations, and transilient turbulence (Stull, 1989) cannot be represented by the TKE closure used here. Nevertheless, it has been found that the simpleTKE closure in conjunction with partially resolved cumulus updrafts created realistic vertically oriented plumes of high TKE which significantly enhance explicitly predicted vertical transport locally. Also, horizontal advection of the TKE acted to spread the effects of cumulus mixing downstream, and in particular, around the eye wall. This can be significant since the time scale of turbulent dissipation was approched by the inertial frequency as the storm increases in strength. This will be shown in the discussion of results below.

The surface fluxes of moisture, sensible, and radiative heat were calculated assuming an ocean of uniform temperature $301 \mathrm{~K}$. The surface layer parameterization was based on the Louis (1979) surface layer, with surface roughness specified as a function of wave height (Delsol et al., 1971).

Horizontal mixing was based on a deformation based 2nd order eddy viscosity closure described by Tripoli and Cotton (1982) with a background fourth order diffusion as described by Klemp and Wilhelmson (1978). The 1.5 level closure was deemed inappropriate for the horizontal because the issues controlling turbulence were more closely tied to numerical as well as physical enstrophy cascade rather than subgrid scale dry and moist convective processes which control vertical diffusion.

Radiative transfer in a cloudy atmosphere was predicted with the radiation parameterization developed by Chen and Cotton (1983). A modified form of the Cotton et al. (1986) (hereafter referred to as CEA) explicit microphysics prediction scheme was employed. The scheme predicts rain, graupel, pristine crystals, and snow crystals. Major modifications made were to:

(1) Divide the original pristine ice category into a snow and pristine category. The modified pristine category was assumed to be composed of newly nucleated hexagonal plate crystals of uniform mass $1.5 \times 10^{-12} \mathrm{~kg}$. This size was determined in separate axisymmetric simulations of a tropical cyclone with explicitly predicted pristine crystal sizes. The new snow category was assumed to follow a Marshall-Palmer 
distribution with slope and intercept derived from an explicitly predicted number concentration per unit mass, similar to the number concentration prediction of CEA. The snow was assumed to have grown from its nucleation size and to be somewhat rimed.

(2) Aggregated crystals were assumed to be part of the snow category. Aggregation of snow and pristine crystals was represented by appropriate transfers of mass to snow from pristine crystals and appropriate adjustments of the predicted snow concentration. The characteristic snow diameter was limited to not exceed $0.33 \times 10^{-2} \mathrm{~m}$, which was consistent with the Cotton et al. (1986) aggregate distribution slope.

The CEA model originally grouped both nucleated and new crystals together. Since a constant size distribution had been assumed, massive nucleation at cold temperatures would drastically alter the average crystal size and would remove all memory of the growth that some of the larger crystals had been through. Here, growth processes were assumed to convert only a given number of crystals at the specified mass to the snow category, which itself was logarithmically distributed. Hence, new and mature populations of crystals would continue to exist where massive nucleation occurred. This was especially important to the simulations of cirrus anvils.

(3) Graupel was represented with a constant slope Marshall-Palmer distribution, with a characteristic diameter of $2 \times 10^{-4} \mathrm{~m}$. This value was derived from explicit axi-symmetric simulations with predicted graupel mixing ratio and concentration where it was found to be characteristic of the graupel size predicted.

The numerical infra-structure of the model was based on a hybrid leap frog (dynamics) and forward (thermodynamics) time integration on advection with a semi-implicit time split approach to representing the high frequency pseudo-sound wave terms. An enstrophy conserving leap frog advection scheme was used for the integration of wind and pressure while a forward 6th order Crowley was used for the integration of the thermodynamical quantities.

A two-way multiply nested Arakawa "C" grid system was employed, using a form of the meshing technique employed by Tremback et al. (1990) and Clark and Farley (1984) modified for a hybrid time differencing scheme and to accommodate a fourth order smoothing operator in the grid center. The grid nesting scheme was also modified to allow any nest to move along a specified trajectory or to move with the surface pressure minimum.

As shown by Tripoli (1992), the model's mean mass field must be specified externally because of the quasi-compressible closure produces a pressure solution unique only to within a constant. This constant was determined by requiring the mean surface pressure across the outer domain to remain constant by making an adjustment to the mean exner function over the entire domain at every large grid time step. The adjustment was made to the exner function so that local hydrostatic balance was unaffected by the change. In addition to this adjustment, the outward and inward changes of mass flux predicted by the external boundary's radiation condition were forced to balance by reducing excessive inflow (or outflow) tendencies on a percentage basis.

\section{Experiment Design}

The prescribed initial horizontally homogeneous basic thermodynamic structure was taken from the temperature and humidity profiles observed at Kingston, Jamaica, 36 hours before the passage of Hurricane Gilbert. Initial mean winds were assumed to be zero.

The grid locations, spacings, lengths, timesteps and implementation period appear in Table 1. The outer grid was centered along the expected path of the modeled storm, while the medium grid was centered over the location of the initial perturbation.

The initial mean state was perturbed by a modified Rankine vortex described in Appendix B. The vortex was defined by its relative vorticity field which was set to decrease from $2 \times 10^{-4}$ near the center, to slightly negative outside the center and then to increase to zero again. The vorticity field is adjusted so that the total area weighted negative vorticity exactly balances the positive vorticity at a radius of $800 \mathrm{~km}$. Hence no net vorticity was added to the flow. The stream function field was formed from the relaxation of the vorticity field which was in turn used to calculate the wind field. 
Table 1. Two-Way Nested Grid Setup

\begin{tabular}{lcccc}
\hline Grid & 1 & 2 & 3 & 4 \\
\hline Period of activation & $0-56 \mathrm{hr}$ & $0-56 \mathrm{hr}$ & $8-56 \mathrm{hr}$ & $50-56 \mathrm{hr}$ \\
\# Central latitude & $17.5 \mathrm{~N}$ & $16.5 \mathrm{~N}$ & $16.5 \mathrm{~N}$ & $18.5 \mathrm{~N}$ \\
\# Central longitude & $71.0 \mathrm{~W}$ & $68.0 \mathrm{~W}$ & $68.0 \mathrm{~W}$ & $69.7 \mathrm{~W}$ \\
\# Zonal boxes & 64 & 60 & 64 & 60 \\
Zonal spacing & $60 \mathrm{~km}$ & $20 \mathrm{~km}$ & $10 \mathrm{~km}$ & $3.3 \mathrm{~km}$ \\
Zonal domain length & $3840 \mathrm{~km}$ & $1200 \mathrm{~km}$ & $640 \mathrm{~km}$ & $198 \mathrm{~km}$ \\
\# Meridional boxes & 64 & 60 & 64 & 60 \\
Meridional spacing & $60 \mathrm{~km}$ & $20 \mathrm{~km}$ & $10 \mathrm{~km}$ & $3.3 \mathrm{~km}$ \\
Meridional domain length & $3840 \mathrm{~km}$ & $1200 \mathrm{~km}$ & $640 \mathrm{~km}$ & $198 \mathrm{~km}$ \\
\# Vertical boxes & 42 & 42 & 42 & 42 \\
Vertical spacing & $400-800 \mathrm{~m}$ & $400-800 \mathrm{~m}$ & $400-800 \mathrm{~m}$ & $400-800 \mathrm{~m}$ \\
Domain height & $26 \mathrm{~km}$ & $26 \mathrm{~km}$ & $26 \mathrm{~km}$ & $26 \mathrm{~km}$ \\
Large timestep & $120 \mathrm{~s}$ & $40 \mathrm{~s}$ & $20 \mathrm{~s}$ & $6.33 \mathrm{~s}$
\end{tabular}

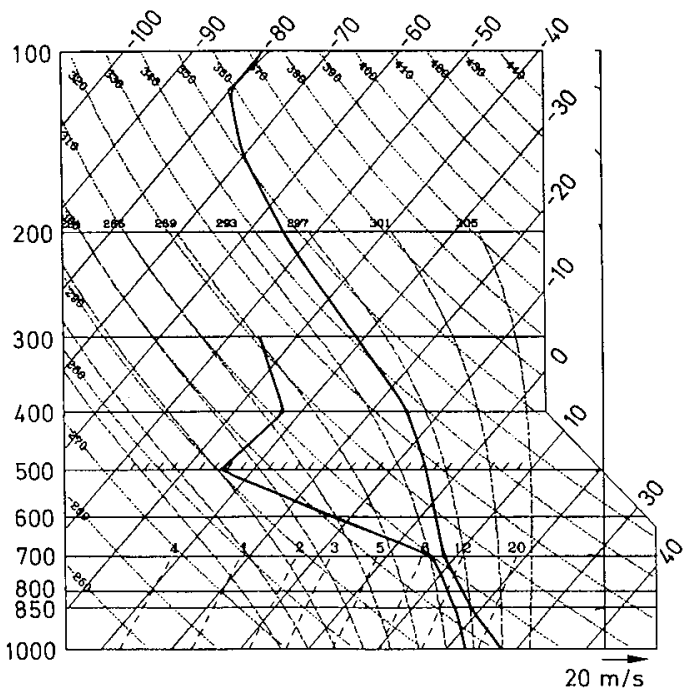

Fig. 1. Initial sounding observed at Kingston, Jamaica at 0000 UTC 11 September, 1988

The perturbation produced a maximum tangential wind of $11 \mathrm{~ms}^{-1}$ at the surface at a radial distance of $150 \mathrm{~km}$ from the vortex center.

Corresponding virtual potential temperature and pressure fields were then calculated using the nonlinear balance equation. The outer grid perturbation pressure field was first found using Neuman boundary conditions and then normalized to the observed sounding at the location of Kingston, Jamaica (Fig. 1). The potential temperature field was determined by assuming hydrostatic balance. The medium mesh was dynamically balanced using Dirchlet boundary conditions on pressure determined from the integration of the outer grid.

The third mesh was not implemented until 8 hours of integration in order to conserve computer resources during the period when the model was beginning to build an Ekman Layer. The fourth grid, with a $3.3 \mathrm{~km}$ horizontal resolution was implemented for a 6 hour period at the mature stage of the simulated tropical cyclone. The limited time of its use was both because of the high cost of its use ( 8 hours of Cray-ymp for 1 hour of simulation), and because the limited horizontal extent of the grid required that the eye wall be contained within a box $200 \mathrm{~km}$ on a side.

\section{Results}

\subsection{Evolution of Structure}

\subsubsection{Initial Rapid Growth Phase - Transition to Tropical Storm}

The simulation was initiated at 0000 LST. Because the initial modified Rankine vortex was specified to be nondivergent, the vortex must have developed a divergent wind component by frictional processes to initiate convective activity. In this section, the modeled transition from a balanced ${ }^{1}$ and nondivergent vortex, to a developing tropical cyclone

\footnotetext{
1 "Balanced" refers to the state where the wind and mass fields are linked to each other so that the time tendency for horizontal divergence vanishes, as dictated by the nonlinear balance equation.
} 

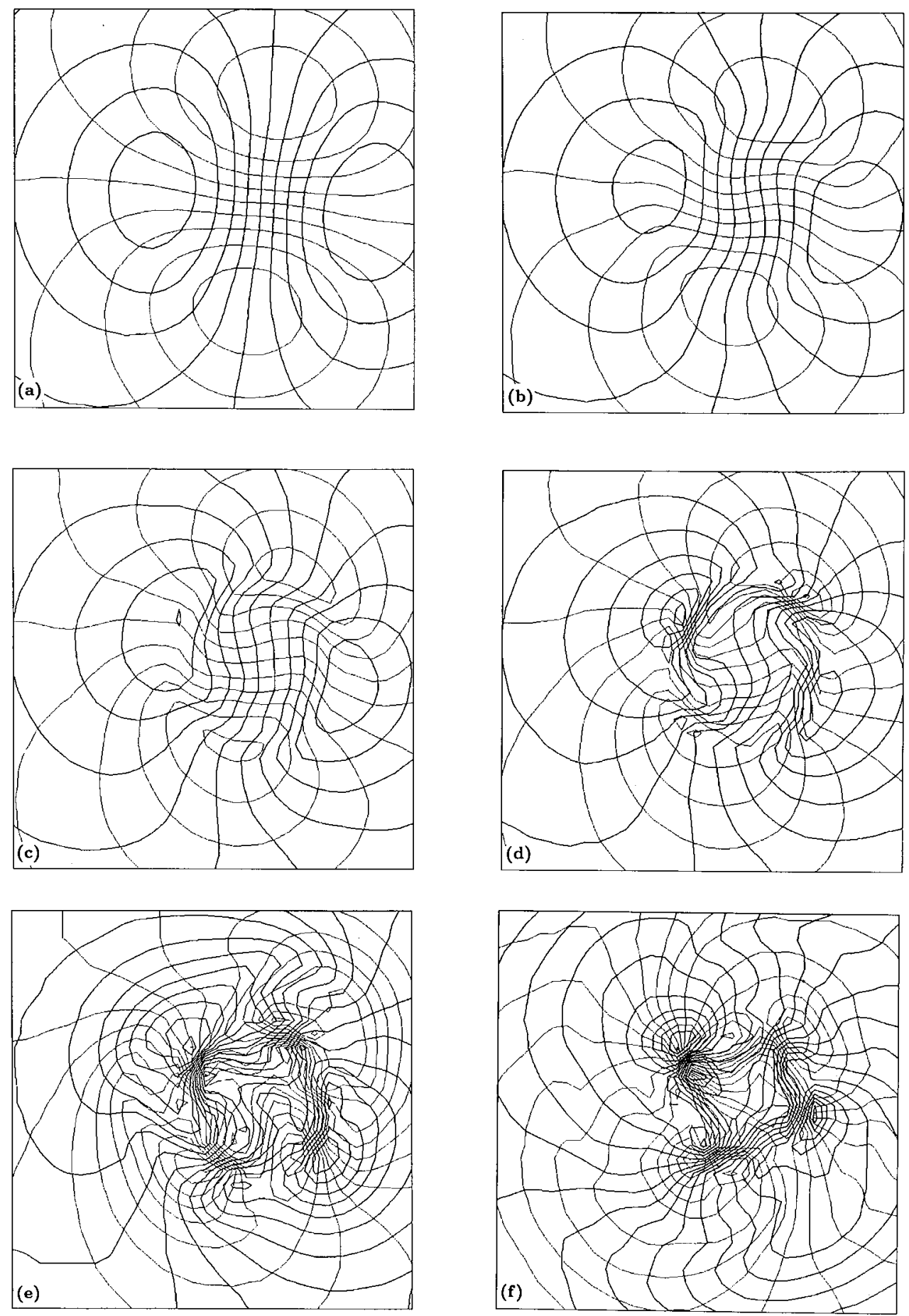

Fig. 2. Contours of zonal $(u)$ and meridional $(v)$ wind components at intervals of $2 \mathrm{~ms}^{-1}$ over a region $600 \mathrm{~km}$ across centered near the vortex core and viewed from the second mesh. Simulation time is (a) $5 \mathrm{hr}$, (b) $7 \mathrm{hr}$, (c) $8 \mathrm{hr}$, (d) $9 \mathrm{hr}$, (e) $10 \mathrm{hr}$ and f) $11 \mathrm{hr}$ of simulation

is described. Admittedly, the modeled transition was strongly dependent on the initial structure of the Rankine vortex, and such transitions in a real developing cyclone were likely to be strongly dependent on the nature of the initial perturbing disturbance and large scale forcing. It was however instructive to look at the modeled process of transition in order to gain some insight into the nature of the explicit interaction between a weakly inertially stable vortex and relatively strong, and 
at times dominant, deep convection processes in three dimensions.

During the first 5 hours of simulation, surface friction gradually weakened the initial tangential wind in the lowest layer causing it to turn slightly inward. Figure $2 \mathrm{a}$ depicts the variation of the zonal and meridional wind components ( $u$ and $v$ respectively) at 5 hours. The intersecting $u$ and $v$ contours formed a grid at the center of rotation. The $u$ and $v$ grid direction, if defined to be the direction of the central $v$ component contour pointing toward negative $u$ values, indicated the relative degrees of convergent and rotational flow. The initial $u$ and $v$ grid direction, with only a cyclonic rotational wind, pointed directly north. By five hours, a convergence was evident by the slight veering of the $u$ and $v$ grid direction.

By 7 hours, it was found that the surface $u$ and $v$ grid in the inner vortex had begun to back, indicative of the development of divergence in the vortex center. This resulted from a maximizing of surface convergence at a radius of $250-300 \mathrm{~km}$ which was roughly equivalent to the radius of peak velocity specified initially for the Rankine vortex. This forced a shallow upward circulation at the radius of maximum convergence, which subsequently forced mass inward, above the surface layer toward the vortex center. That, in turn, caused the surface pressure in the center to rise slightly, reducing the horizontal pressure gradient at the center. As a result, the flow inside the convergence ring became unbalanced and began to diverge outward, strengthening the convergence.

As the convergence sharpened, a circular ring of condensate formed from air being forced upward to the lowest condensation level (LCL). Peak vertical motions grew very slowly at this time before the moisture reached the level of free convection (LFC). By 8 hours, Fig. 2c showed an amplified convergence ring. As a result of inward angular momentum transport, peak winds had increased slightly and regained most of the speed lost to friction earlier.

Shortly after 9 hours (Fig. 2d) the forced cloud ring reached the $L F C$ and the vertical velocities began to dramatically increase from magnitudes of centimeters per second to meters per second. The deepening convection warmed the air at mid to upper levels, and resulted in a more rapid lowering of the surface pressure which greatly enhanced the surface convergence into the ring and hence the vertical growth rate of the ring as well.

In general, because convection favors the smallest scales in both the radial and tangential directions, any asymmetric perturbation will likely initiate a tangential variation of the convection. In this otherwise symmetric initial perturbation, the only asymmetry, besides the beta effect, was the tangential variation of the finite differencing truncation error. This had a distinctly tangential wave number four variation due to the rectangular grid geometry. This very small tangential perturbation grew rapidly and by 10 hours (Fig. 2e), a distinct pattern of four dominant convective centers appeared, with some weaker meridional variation of intensity superimposed from the beta effect.

The role of convection in forcing surface convergence became dominant over the frictional effects by this time. As a result, the surface velocity fields converged into these convective systems to the point that the flow in the major vortex center actually became anticyclonic while the four convective centers each developed closed circulations of their own. In fact, at 10 hours, the surface lows beneath the convective systems reached a perturbation pressure (perturbation from the horizontal mean) of $-5.5 \mathrm{hPa}$ while the central pressure of the parent vortex pressure was $-3.5 \mathrm{mb}$. This was $0.5 \mathrm{hPa}$ greater than the initial surface pressure. Hence, the initial modified Rankine vortex had broken up into four separate meso- $\beta$ scale convective systems.

Simulated precipitation formed within the individual convective systems and, as with any unbalanced convective system, maturity was reached and the system progressed into a breakdown phase (Tripoli and Cotton, 1989). This resulted in the formation of deep internal waves radiating from the center of the collapse. Phase speeds were calculated from animated sequences of the vertical motion field and found to be about $35 \mathrm{~ms}^{-1}$. The collapse also resulted in the rise of the surface pressure beneath the convective centers. Convergence, forced at upper levels within the parent vortex center, resulted in a strong surface pressure decrease to $-11 \mathrm{hPa}$ by 11 hours (Fig. $2 \mathrm{f}$ ). Also evident were outward propagating waves in the $u$ and $v$ fields indicated by the wave like structures in the contours away from the center. Three dimensional visualization of these waves 


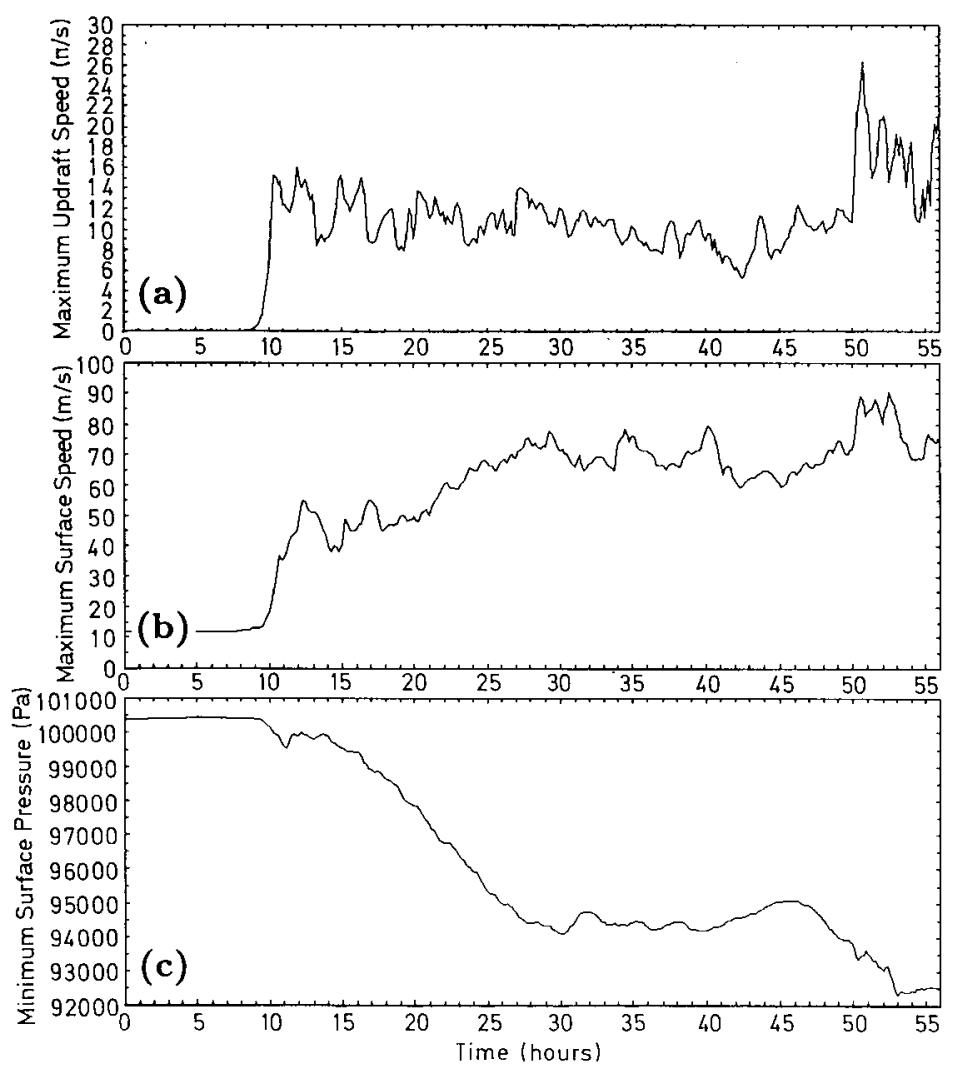

Fig. 3. Graphs of (a) peak updraft, (b) peak wind speed and (c) minimum surface pressure over the storm integration volume of the finest grid activated as a function of time from 0 to $56 \mathrm{hr}$ (not shown for this particular time) showed these waves to be spiral bands of vertical motion, not associated with deep convection outside the inner convergence ring. The bands sloped upward and outward, indicative of upward and outward propagation (Durran, 1981).

It must be recognized that the precise number and sizes of the mesoscale convective systems formed were probably greatly affected by the initiating mechanism from which they were created. In this simulation it was the tangential variation resulting from numerical truncation that caused the four cell convective system structure. The model grid resolution of the simulation was also an important factor, but not thought to be the dominant one since the meso- $\beta$ scale systems were over 15 grid lengths across and so not near the smallest scale possible. It is most reasonable to expect that in a more realistic scenario, the convection would have developed a strong tangential variation as it did here, although the size and number of systems would have been dependent on variations in initial conditions. It is also likely that given no radial variation initially, there is an optimal size and number of convective systems that would form. Nevertheless, these results suggested that even minor departures from axisymmetry would take precedence over any tendency for an optimal size in determining the scale of the initial convective systems forming in the vortex. It is perhaps unsettling to this study that there is no reason to believe that this would not have a significant impact on the evolution of the cyclone.

After the 11 hour integration time, another period of meso- $\beta$ scale growth ensued. Here, the low pressure again became most intense beneath three primary convective systems, reaching $-7.5 \mathrm{hPa}$ between the most intense system, compared to $-6.5 \mathrm{hPa}$ beneath the parent vortex central pressure. Note, however, that the parent vortex had closed the gap between its intensity and that of the meso- $\beta$ scale convective systems of the first growth period. This was a result of the growth of the slow manifold ${ }^{2}$ in response to longterm inertial adjustment to the convective heating.

\footnotetext{
${ }^{2}$ The slow manifold here reverses to the balanced part of the system, which evolves with inertial frequencies as opposed to fast manifold modes which evolve with frequencies related to the Brunt Vaisala frequency.
} 
The growth and decay cycle of mesoscale convective system (MCS) activity surrounding the cyclone core continued for several hours. During this time, the amplitude of the slow manifold increased in strength and eventually reached the point where it could maintain inflow over and above the modulated inflow and outflow of the surrrounding convective systems. This occurred between 16 and 18 hours of simulation. This pulsating development process was also shown by Tripoli and Cotton (1989) to be an important component of the organization of orogenic mesoscale convective systems and could be viewed as the realization of the geostrophic adjustment process described theoretically by Schubert and Hack (1982).

During this period of growth which began at 9 hours of simulation, a rapid acceleration of the tangential wind occurred. Figure 3 shows the evolution of peak vertical motion, peak horizontal wind gust, and lowest surface pressure. The peak wind gust was somewhat less related to the tangential wind during the early stages because of the strong divergent wind in the early stages of storm development. Sustained tangential winds were found to be near $15 \mathrm{~ms}^{-1}$ at 11 hours and $20 \mathrm{~ms}^{-1}$ at 13 hours and $22 \mathrm{~ms}^{-1}$ at 14 hours. Hence, the tropical storm phase was reached around 14 hours of simulation, approximately 5 hours after the first free convection. The surface low at the center of the storm vortex was $-8.5 \mathrm{hPa}$ perturbation pressure of $1002 \mathrm{mb}$. Since the 14 hour time was actually in a peak MCS phase, the surface pressure was actually $0.5 \mathrm{hPa}$ higher in the center than it was at 13 hours. Nevertheless, the 14 hour time marked the first point where the surface pressure remained equal to or lower than the MCS pressure lows surrounding the vortex center during the MCS phase.

Figure 4 shows the microphysical, TKE, $\theta_{V}^{\prime}$, vertical motion (viewed from below to see the low-level vertical motion), $\theta_{e}$ and potential vorticity at 14 hours. The Ertel potential vorticity $(Z)$ used is defined as:

$Z=\rho \eta \cdot \nabla \theta$

where $\rho$ is air density, $\eta$ is the absolute vorticity vector and $\theta$ is the potential temperature. The view is from the 2 nd grid nest in order to attain a storm scale perspective.
Note that at that time, spiral bands were already apparent in the cloud water field. Precipitation was evident beneath the developing storm. At that time, a cirrus anvil was in the initial stages of formation. There was a false eye within the condensate field resulting more from the absence of convective towers than from forced subsidence. This can be seen from the lack of a warm core in the $\theta_{v} l$.

The microphysics of the convective regions were dominated by pristine crystals at $12-15 \mathrm{~km}$, snow at $5-12 \mathrm{~km}$, graupel at 5-7 km AGL, and rain below the melting level. Primary precipitation growth occurred by the Bergeron-Findeisen process followed by riming within the supercooled liquid water to produce graupel. The graupel then melted and formed rain. The snow, composed primarily of aggregates and rimed crystals reached average water contents in excess of $0.6 \times 10^{-6}$ $\mathrm{g} \mathrm{m}^{-3}$ at $10 \mathrm{~km} \mathrm{AGL} \mathrm{(above} \mathrm{ground} \mathrm{level).} \mathrm{Graupel}$ reached contents near $2 \times 10^{-6} \mathrm{~g} \mathrm{~m}^{-3}$ and melted entirely before reaching $3 \mathrm{~km}$ AGL. The massive melting between 3 and $5 \mathrm{~km}$ AGL was found to have a major impact on the entire storm evolution and will be discussed in more detail below. Rain reached contents near $5 \times 10^{-6} \mathrm{~g} \mathrm{~m}^{-3}$ from collision coalescence growth. This produced peak rainfall rates of over $9 \mathrm{~cm}$ per hour at the surface.

Figure 4 also shows that the TKE field formed vertically erect plumes of vertical mixing in the vicinity of the deep convective towers. The TKE reached peak magnitudes of $10-20 \mathrm{~m}^{2} \mathrm{~s}^{-2}$, showing that considerable vertical mixing assisted the explicit vertical transport. Nevertheless, peak vertical velocities within explicit convective motions were reaching $6-12 \mathrm{~ms}^{-1}$ at that time. This was large considering the meso- $\beta$ scale of the fine mesh.

Vertically propagating internal waves within the stratosphere were evident in the $\theta_{v}^{\prime}$ field. Animated visualization of the development showed that they emanated from overshooting updrafts and were spawned in a spiral with an angular frequency at the center equal to the angular frequency of the cyclone. This frequency was related to the combined lifecycle of the elevated cloudtop and the time it took to pass a point while circling the storm core. The outward propagation phase speed was $35 \mathrm{~ms}^{-1}$, calculated by clocking the wave front through a 40 minute animated propagation sequence. The vertical wavelength of 

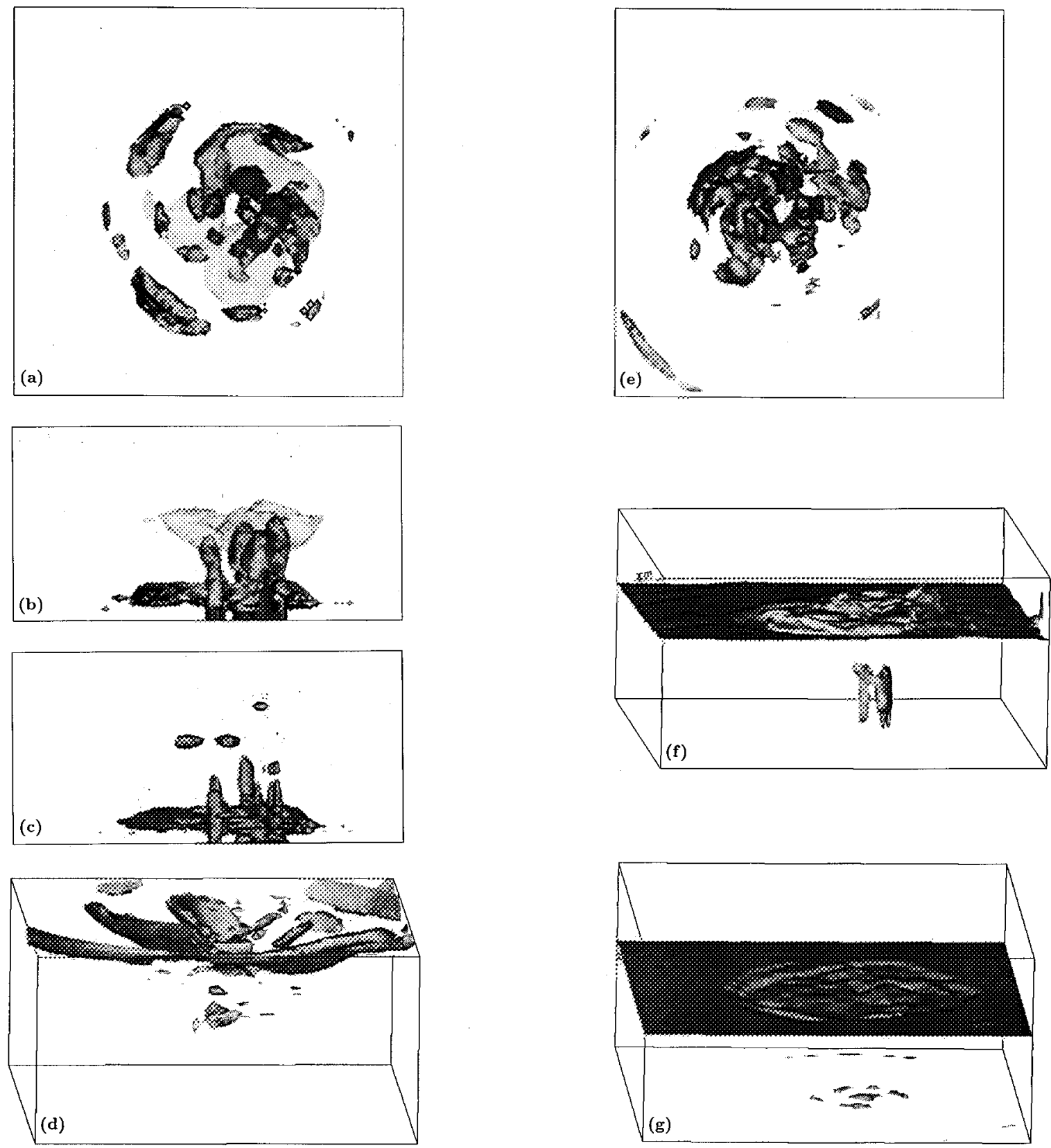

Fig. 4. The simulated three-dimensional storm viewed over the entire second grid (region is $1200 \mathrm{~km}$ across) and at $14 \mathrm{hr}$ showing: (a) and overhead view of the $0.01 \times 10^{-6} \mathrm{~g} \mathrm{~m}^{-3}$ condensate surfaces with north pointing up (the rain and graupel surfaces are darkely shaded, the cloud water and snow surfaces are lightly shaded, and the pristine crystal surface is partially transparent), (b) the view of the condensate surfaces described in a) from the south, (c) the view of the $2 \mathrm{~m}^{2} \mathrm{~s}^{2}$ TKE surface from the south, (d) an oblique view of the $5.1 \mathrm{~K} \theta_{v}^{\prime}$ surface from the west, (e) oblique view of the $0.3 \mathrm{~ms}^{-1}$ (lightly shaded) and $-0.3 \mathrm{~ms}^{-1}$ (darkly shaded) vertical motion surface from below with north facing up (note east is to the left here) (f) oblique view of the 0.5 PVU surface from the south, and $(\mathrm{g})$ an oblique view of the $355 \mathrm{k} \theta_{e}$ surface from the south

the internal waves was about $9 \mathrm{~km}$ while the horizontal wave length was approximately $150 \mathrm{~km}$. Defining the linear gravity phase speed of:

$c_{p}=\frac{N}{l}$ where $N$ is the Brunt Vaisala frequency of the stratosphere calculated to be $2.4 \times 10^{-2}$ and $l$ is the vertical wavenumber given by:

$$
l=\frac{2 \pi}{L_{z}}
$$



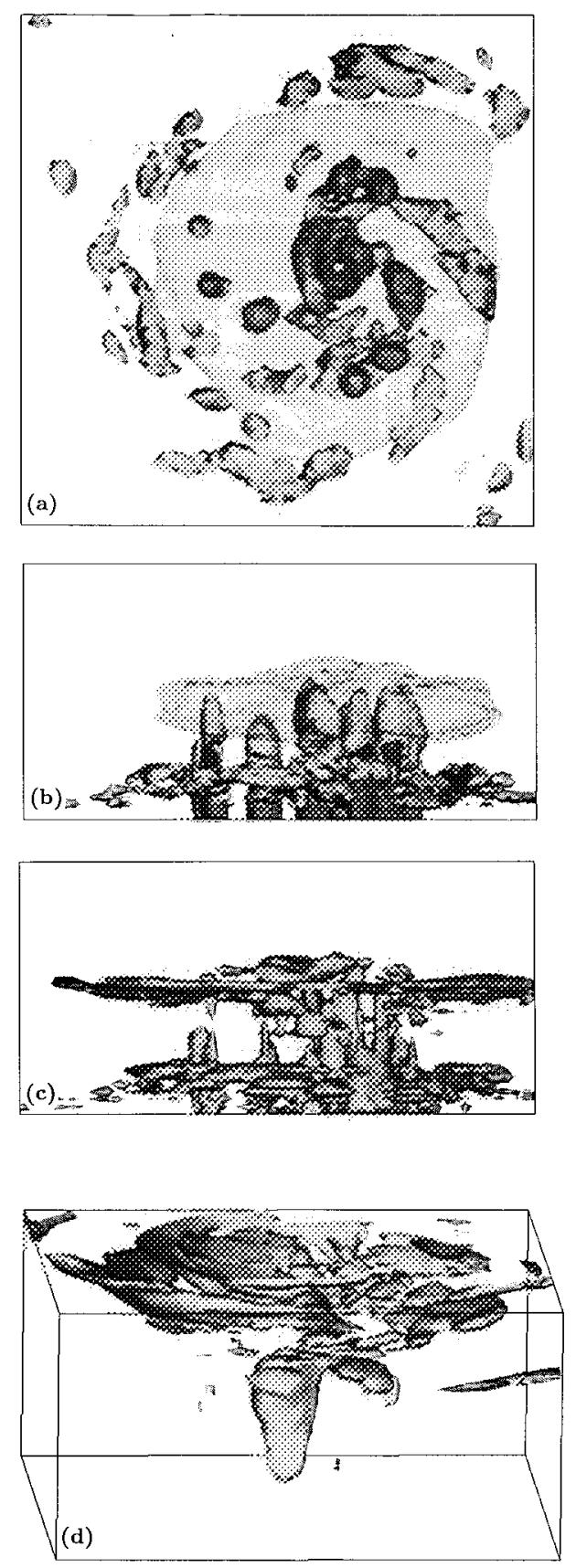

Fig. 5. Same as Fig. 4, except for $26 \mathrm{hr}$ of integration

and $L_{z}$ is the vertical wavelength, then $c_{p}=34.6$ $\mathrm{ms}^{-1}$, which is precisely the simulated phase speed.

The vertical motion field, viewed from below, showed the geometry of both the vertical motion associated with the MCSs and that associated with the gravity waves at all vertical levels. Some spiral bands were visible aloft in this figure, associated with the propagating stratospheric
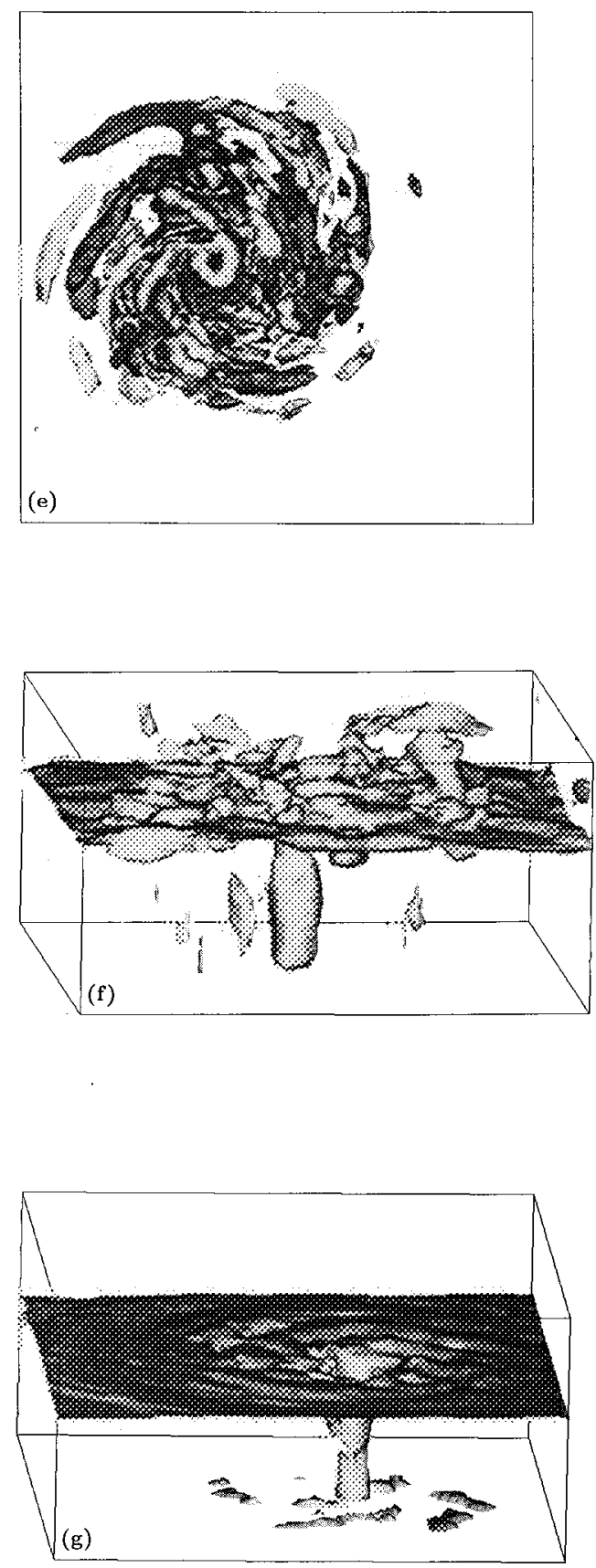

waves. Deep vertical plumes of vertical motion in excess of $1 \mathrm{~ms}^{-1}$, not depicted in this figure existed in association with the MCS activity.

The potential vorticity field showed a small mid-tropospheric maximum had begun to develop in association with diabatic sources within the convection at 14 hours. Finally, the $\theta_{e}$ field showed growth of the $355 \mathrm{~K}$ surface, near the ocean surface, but not yet a consistent vertical plume of 
the $355 \mathrm{~K}$ surface on the scale of the $20 \mathrm{~km}$ averaged grid. The peak $\theta_{e}$ at the surface roughly coincided with the strongest surface winds and hence the strongest moisture fluxes resulting from the bulk surface flux formulation.

\subsubsection{Development of Inertially Stable Vortex Center - Transition to Hurricane}

Figure 5 depicts the storm structure at 26 hours as the first rapidly deepening phase (which began just after 14 hours of simulation) was concluding. The local time was 0200 LST. By that time the surface pressure had decreased to $950 \mathrm{mb}$, for a net drop of $52 \mathrm{hPa}$ in the $12 \mathrm{hr}$ period since the 14 hour time discussed earlier. Peak horizontal winds had increased to average speeds approaching $70 \mathrm{~ms}^{-1}$, clearly reaching the hurricane intensity range.

The condensate field showed that the convective activity had greatly spread outward from the eye wall in a banded structure. Low elevation liquid only stratiform cloudiness was associated, with much of the banded cloudiness, showing that the clouds were forced by the bands rather than the clouds forcing the bands.

The vertical motion field exhibited a good eye wall within the troposphere with a series of spiral vertical motion bands radiating outward from the center at all levels. The spiral bands of vertical motion were most apparent within the stratosphere and had amplitudes of 0.25 to $0.5 \mathrm{~ms}^{-1}$. Over approximately $30 \%$ of the region, however, the stratospheric bands extended downward into the troposphere, where a second vertical motion maximum was found approximately one vertical wavelength below the stratospheric maximum. The spiral bands were associated with a temperature perturbation of $5-10 \mathrm{~K}$ amplitude in the stratosphere and less than $2 \mathrm{~K}$ in the troposphere.

Over much of the region, the bands extending into the lower troposphere were coincident with the condensate discussed above. Animated visualization of the condensate field and vertical velocity field demonstrated that the condensate field was not moving outward with the waves. Instead, the waves tended to organize and amplify existing cloudiness as they propagated through the cloudiness. The clouds within the bands, tended to be short lived when compared to the propagating bands. This made individual groupings of clouds impossible to track for more than a couple of hours. Over the period they did exist, however, and they seemed to spiral slightly inward, with the low level flow. In this sense, they behaved similarly to the mesoscale convective systems at earlier times.

The outlying regions of cloudiness were found to have a significant impact on the surface layer $\theta_{e}$ field, creating relatively cold pockets. It was found that these cold pockets grew strongest from the melting of graupel at the melting level. In fact, in at least one case, a $\theta_{e}$ minimum was formed at the surface from melting above, which was less than the environmental minimum. These theta minima proved to be long-lived and subsequently worked their way into the eye wall. Moreover, the stronger maximum seemed to initiate new convection when intersecting with outward propagating waves. As these density currents became caught up in the vortex, they tended to elongate in the horizontally sheared flow and become band-like themselves.

The surface beneath regions of convection within the eye wall was found to be associated with a relative $\theta_{e}$ minimum itself for the reasons discussed above for the outer bands. This produced a mild density current and a local pressure increase at the surface. This undoubtedly enhanced the convergence into the eye wall. Moreover these density currents together with the density currents merging from the outer bands enhanced the asymmetry of the eye wall, sometimes resulting in deep convection over only half of the eye wall. In fact, it was rare to have a completely closed pattern of heavy surface rain beneath the eye wall.

As these density currents moved around the eye wall, they seemingly split off the outer edges of the surface pressure gradient field, giving rise to the outwardly propagating bands which were distinctly visible in animated sequences of surface pressure. Since the outward propagating bands were also closely connected with the stratospheric forcing, the split in surface pressure gradient occurring at the formation of the band was inherently coupled to the stratospheric formation associated with the moving elevated cloudtop.

The predicted TKE structure showed that there was a continued existence of deep mixing processes in support of the crudely resolved explicit deep convection. The TKE within the eye wall had begun to form a closed cylinder in the lower portion of the eye wall, with individual plume 
structures remaining aloft. This was indicative of the emphasis of storm scale spiraling eye wall convection in the lower levels. This resulted from increasing inertial stability which suppressed the development of divergent wind in response to the heating in favor of a rotational flow. Note also the extensive anvil level canopy of high TKE produced as a result of both shear production from the outflow layer and byoyant production from radiative cooling at the anvil top.

The $\theta_{v}^{\prime}$ field showed clear evidence of a warm core by 26 hours, forced by subsidence from the stratosphere. The development of the warm core was associated with the cessation of the growth and breakdown process of MCS activity in the eye wall discussed earlier. Prior to the development of sufficient inertial stability of the vortex, the subsidence warming forced by individual convective plumes resulted in the breakdown of the convection in the eye wall due to the destruction of low level pressure gradients supporting the MCS inflow. As the storm scale vortex increased in inertial stability, inertial frequencies increased to the point where the tangential wind of the vortex could adjust to the warming so that divergent flow from the eye wall into the eye would not form and act to destroy eye wall convection. Hence by 18 hours of simulation, the individual plumes ceased their periodic breakdown in the eye wall, and the growth of a persistent warm core commenced.

This was shown clearly by Fig. 6 which depicts a series of trajectories at the early stage of storm development centered around 14 hours and the maturing stage centered around 26 hours. Note that in the early stage, the updraft trajectories spiraled into the center at the surface and then moved nearly straight up in deep convection plumes. At the later time (Fig. 6b), the trajectories spiraled upward in the lowest $5 \mathrm{~km}$ and then moved vertically upward. As the storm increased in intensity toward 50 hours (Fig. 6c) the spiral became deeper with little or no explicit vertical movement suggestive of vertical convection. Instead of spiral convection dominated, which was indicative of the dominance of the inertial frequency over the convective and gravity wave frequencies.

The continued growth of the inertially stable vortex was also evident by the formation of a strong potential vorticity maximum of up to 2 PVU at the storm core. A strongly varying potential vorticity field was induced in the lower stratosphere
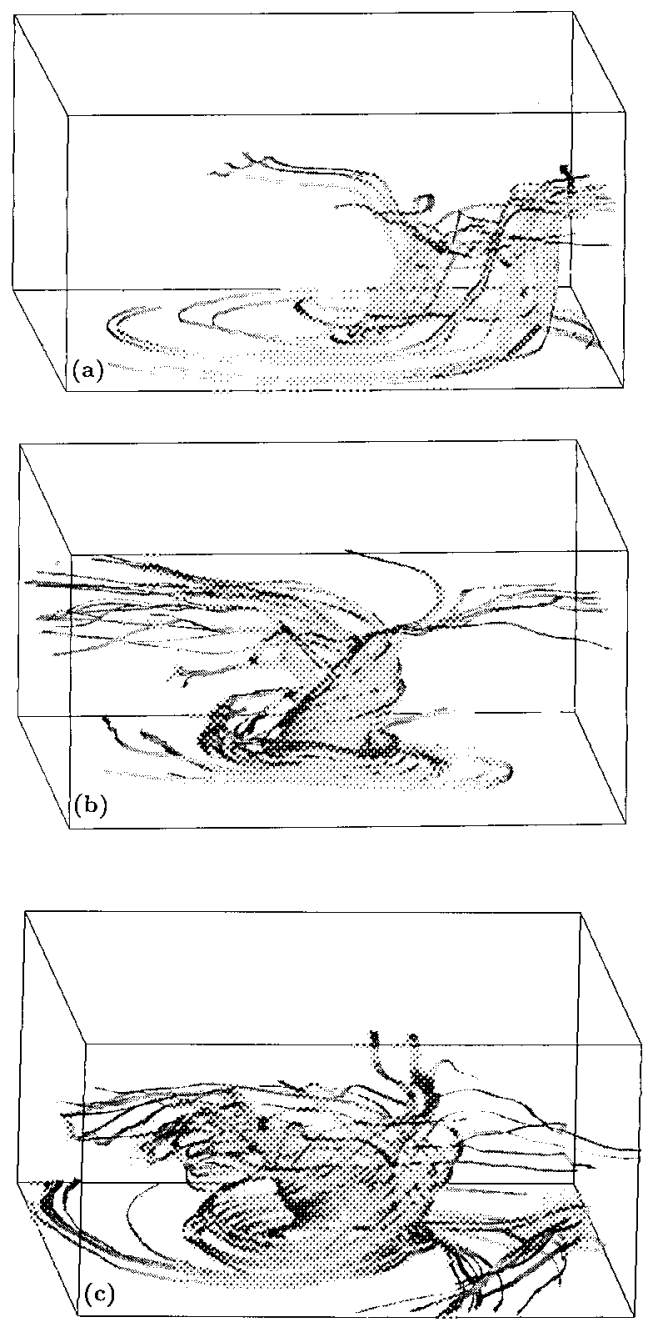

Fig. 6. Oblique view of air trajectories through storm core, viewed from the south centered at (a) $14 \mathrm{hr}$, (b) $38 \mathrm{hr}$ and (c) $48 \mathrm{hr}$ of integration time

by the penetration of convection from below. The potential vorticity structure at the tropopause was characterized by a core of high positive potential vorticity surrounded by regions of negative potential vorticity induced by convective momentum transport from below.

At this time, the $355 \theta_{e}$ surface had formed a vertical conduit within the storm core and from the surface to the stratosphere. The increased surface values of $\theta_{e}$ were distinctly banded, and associated with the banded structure of the cloudiness and vertical motion field.

The increased inertial stabilization of the eye wall was clearly evident in the evolution of the surface pressure and wind vector fields displayed in Figs. 7 and 8. At 14 hours of simulation, 

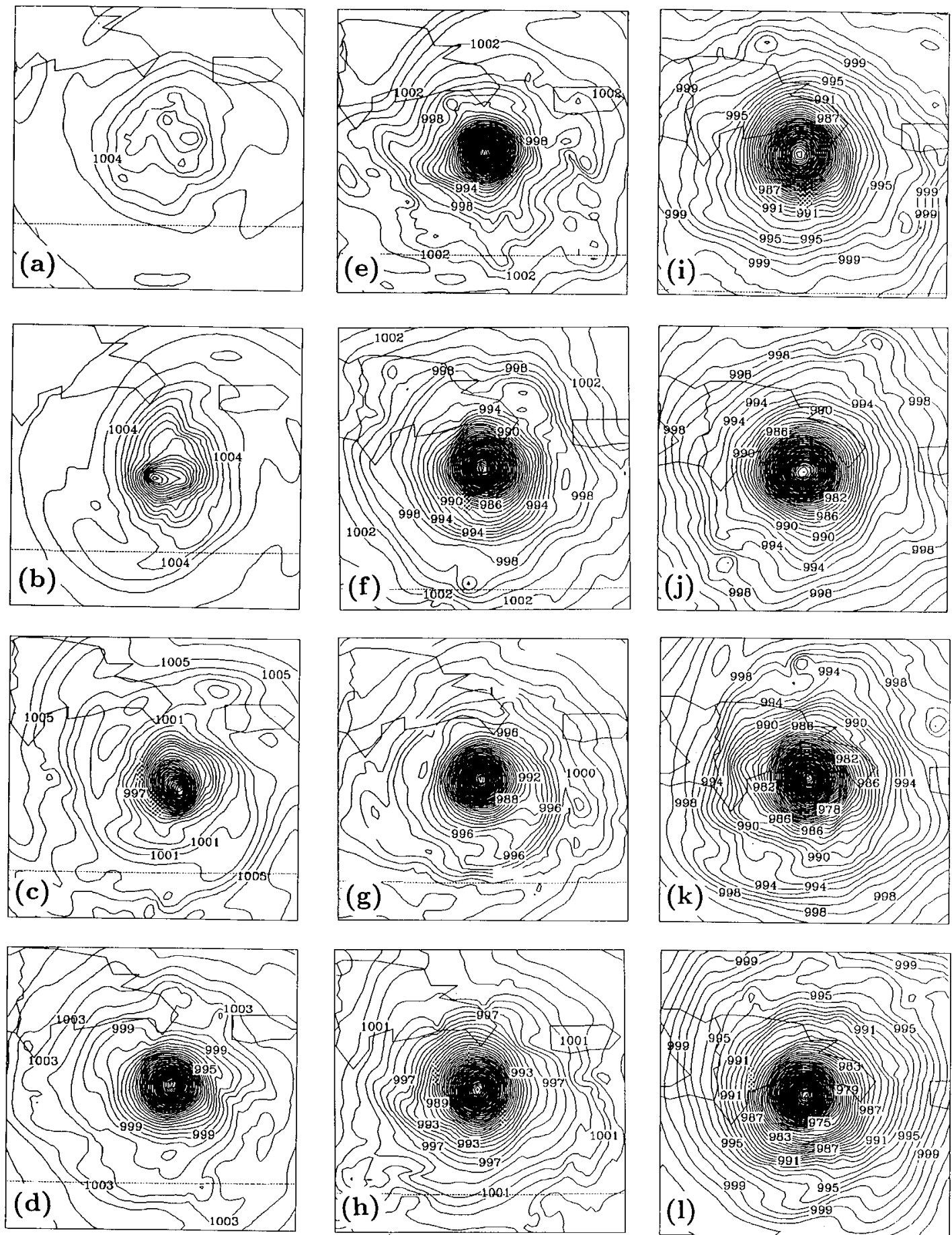

Fig. 7. Surface pressure field contoured at intervals of $1 \mathrm{hPa}$ every four hours beginning at $14 \mathrm{hr}$ of integration with (a) and ending at $54 \mathrm{hr}$ with $(\mathrm{k})$. The pressure at $56 \mathrm{hr}(\mathrm{l})$ is shown

multiple pressure centers were found, while the velocity field was characterized by a series of minor vortices within the primary circulation. By 18 hours a dominant storm core low pressure had emerged and by 22 hours a nearly axi-symmetric low pressure core was evident. At the same time a convergent eye wall flow became more visibly consolidated into a circular convergence band and by 26 hours it became reduced in size to approximately $150 \mathrm{~km}$ in diameter. 

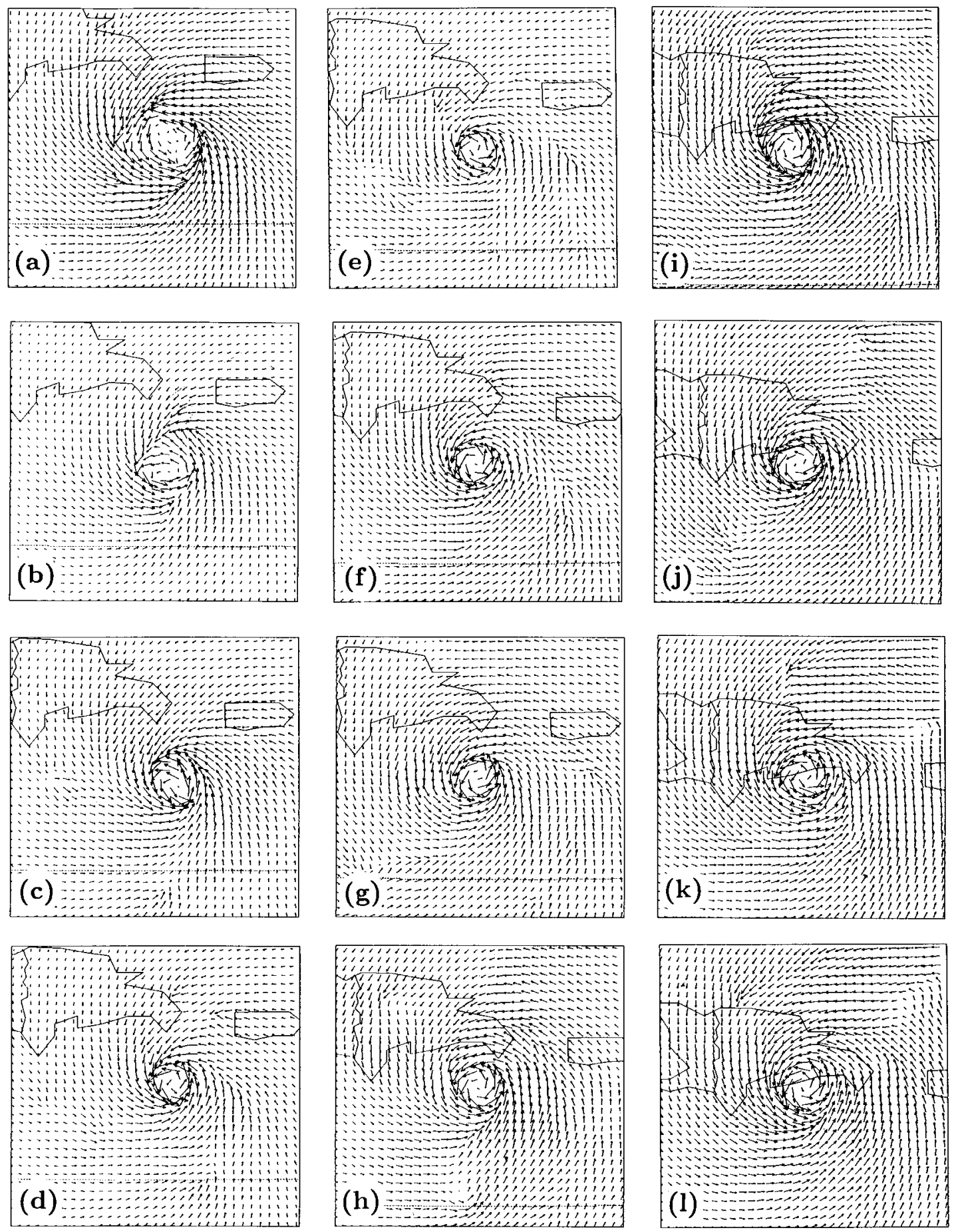

Fig. 8. Same as Fig. 7 except wind vectors are shown instead of pressure at surface level

Outside the storm core, the tangential variation of the pressure structure remained. It was associated with the movement of gravity waves and with regions of convection where density currents were formed. With each major wave node emanating from the core, the rate of pressure fall was momentarily reduced, suggesting that the gravity wave activity was weakening the warm core, and hence reducing the rate at which the cyclone could deepen. Thus, the convection heating was balanced inertially within the core, but continued to form wave activity outside the core where inertial 

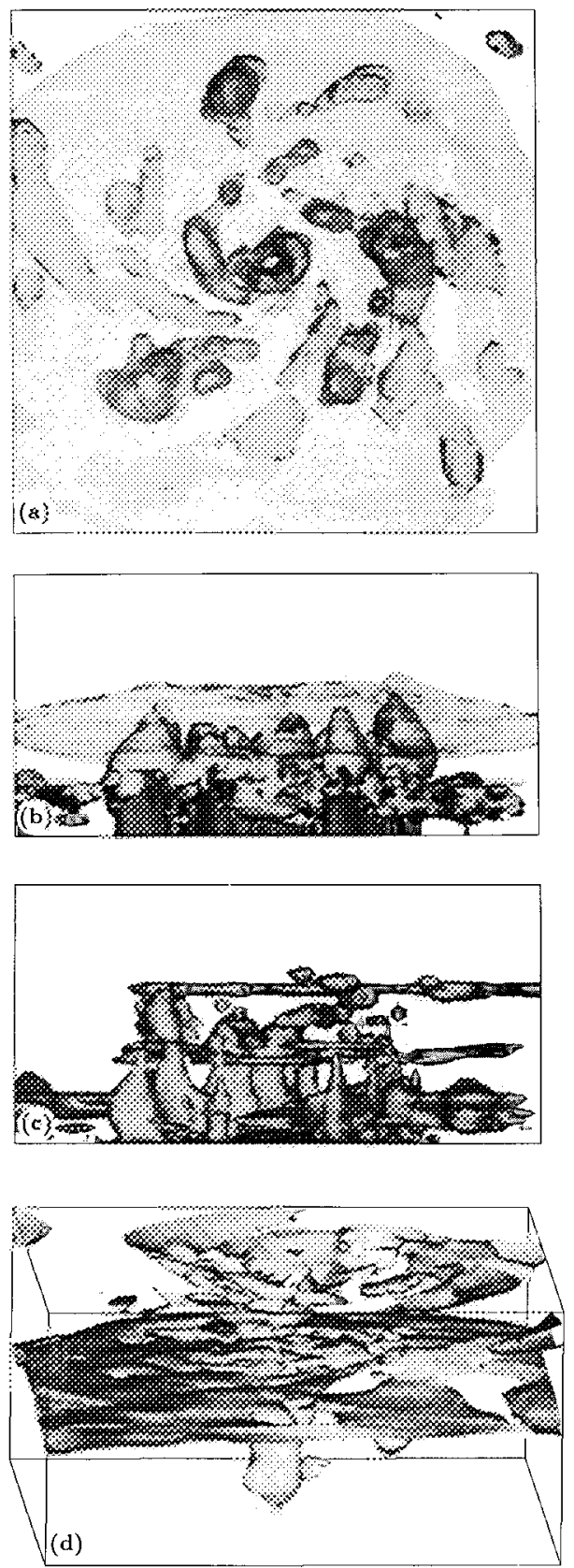

Fig. 9. Same as Fig. 4, except for $38 \mathrm{hr}$ of integration

stability was less. This is again consistent with adjustment theory posed by Schubert and Hack (1982) (Schubert and Hack, 1982).

\subsubsection{Inertially Stable Vortex Attains Quasi-Steady Balanced State at 38 Hours - Hurricane Stage}

The three dimensional storm structure at 38 hours (1400 LST) is depicted in Fig. 9. At this time the
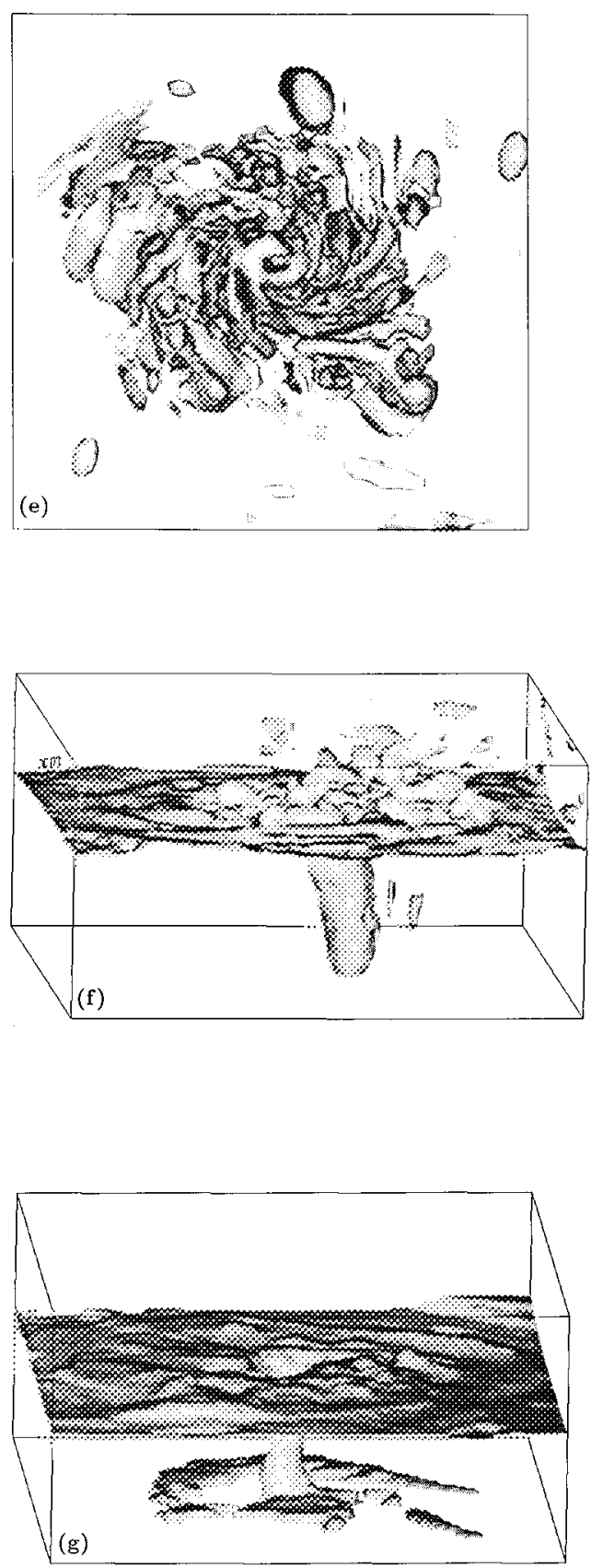

minimum surface pressure had fallen to $942 \mathrm{mb}$, or another $8 \mathrm{mb}$ since the 26 hour time. In fact, all of the pressure fall was before 30 hours when the sun rose above the horizon. Overall, the pressure evolution was much less dramatic than the previous 12 hours and, the times of maximum pressure fall, roughly coincided with darkness.

At the 38 hour analysis time the cirrus anvil, composed of pristine crystals with contents of about $0.5 \mathrm{gm}^{-3}$, had expanded beyond the second 
grid. The vertical motion continued to maintain a circular eye wall, however, the banded structure outside the eye wall had become somewhat fragmented. This seemed to be a result of the convection in the outer bands creating gravity waves of its own, interfering with the more coherent waves emanating from the eye wall.

Systematic warming of the anvil top in the afternoon hours was evident in the $\theta_{v}^{\prime}$ field. It was also evidenced by a weaker TKE maximum at the upper surface of the anvil and the more shallow penetration of the TKE field due to increased stability aloft . The lesser vertical penetration of convection was also evident in the potential vorticity field, which seemed to produce less excitation of stratosphere turbulence than earlier.

Significant outlying convective activity remained, as evidenced by the condensate fields. Comparison of this numerical simulation with a host of previous similar simulations, suggested that much of the outlying convection was supported by the anvil itself. It has been found that the amount of outlying convection was quite sensitive to the amount of water carried out from the center in the anvil. The precise reason why has not been investigated at this time, however, it is speculated that the precipitation may have moistened and destabilized the low to mid levels of the troposphere, releasing the thermal "cap" imposed on outlying convection created by forced subsidence. This enabled the spiral inertia-gravity waves emanating from the center to initiate convection more readily. In fact, in one simulation similar to the one described in this paper except that there was no radiation and there was a slightly different setting on pristine crystal size, the anvil size was dramatically reduced and almost all of the outlying convection was eliminated. This resulted in long term dramatically coherent stratospheric bands over the entire 50 hours of simulation. It is likely that result was at least partially a result of the radiation difference and remains a topic for future research.

Associated with inertial stabilization at 38 hours was the substantial weakening of peak convective updrafts to $6-10 \mathrm{~ms}^{-1}$. This was likely due at least partially to the inertial stabilization of the vortex, although outlying convective updrafts would not be expected to decrease for that reason. Another probable reason was the diurnal stabilization of the upper troposphere in response to daytime warming, which has already been suspected to have weakened the TKE and potential vorticity fields. The fact that the peak updraft and pressure falls increased again after the next sunset, seem to support this hypothesis.

\subsubsection{Explosive Deepening Resumes After Sunset}

Shortly after sunset, which was at 42 hours of simulation (1800 LST), strong deepening of the storm resumed and the central pressure fell from $952 \mathrm{mb}$ at 45 hours to $922 \mathrm{hPa}$ at 53 hours for a total fall of $85 \mathrm{hPa}$ from the initial vortex specification.

The simulation at 50 hours was depicted in Fig. 10. At this time a massive "tree trunk" of $355 \mathrm{~K} \theta_{e}$ rose upward within the eye wall. Convection was more limited to the eye wall and inner bands.

TKE field again began to penetrate into the lower stratosphere as they did at the 28 hour analysis time, although there was an absence of strong TKE along the upper surface of the cirrus anvil, suggesting increased stability over the previous night. It is expected that this may have been a result of the accumulated warming of the region during the day, the effects of which remained.

The warm core remained strong, but was connected to strong warming spread along the base of the anvil as a result of longwave absorption of upwelling radiation from low levels. The potential vorticity maximum continued to be maintained also at 50 hours, although greater perturbation of the stratospheric potential vorticity field again commenced in response to the deepened convection.

The eye wall diameter remained at $120 \mathrm{~km}$, with an eye diameter of about $30 \mathrm{~km}$. Peak tangential winds increased in response to the new growth to $80 \mathrm{~ms}^{-1}$ by 50 hours of integration.

\subsubsection{Extended Simulation with Meso- $\gamma$ Scale Resolution}

Because the simulated eye wall had stabilized at a mean diameter less than $200 \mathrm{~km}$, it was deemed deemed feasible to add a fourth grid with $3.3 \mathrm{~km}$ horizontal resolution to better resolve eye wall processes. The grid was added at 50 hours, and the integration was extended to 56 hours. The vertical motion field shown in Fig. 10 shows that the peak vertical velocities responded immediately to the increase resolution but then tended back 

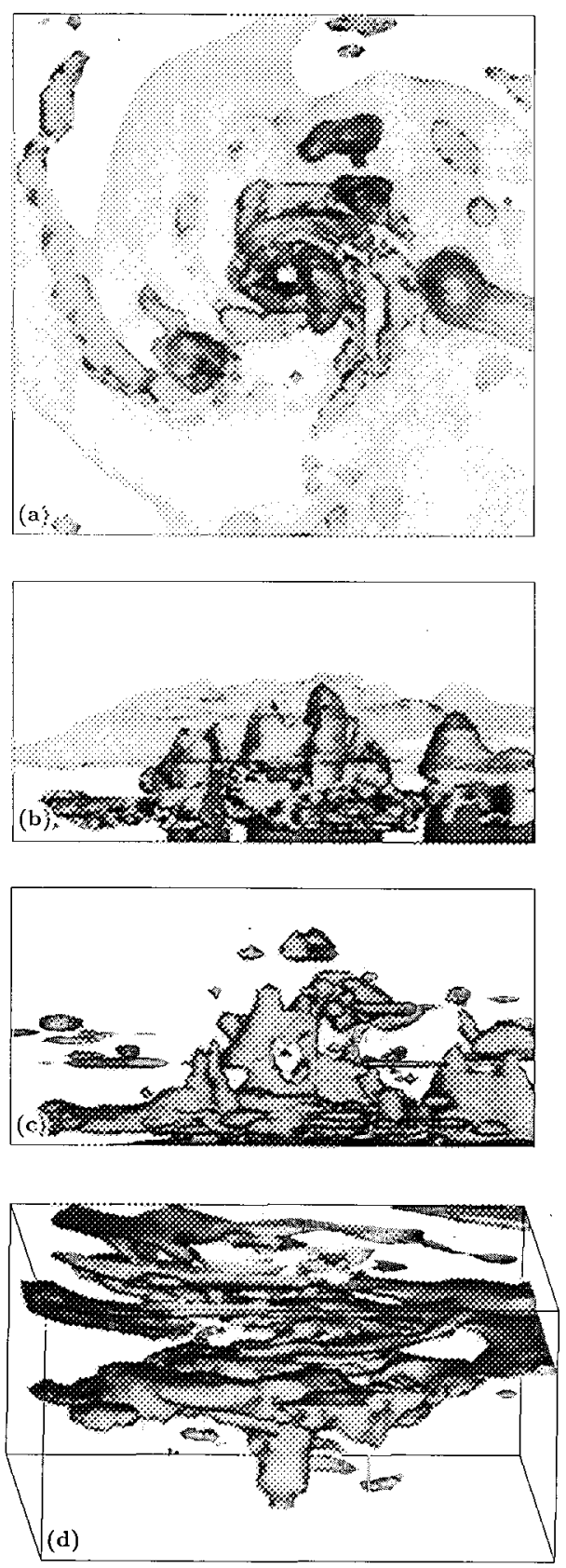

Fig. 10. Same as Fig. 4, except for $50 \mathrm{hr}$ of integration

downward. This probably resulted from a short term imbalance of the core resulting from less overall horizontal dissipation in the balance as a result of less numerically related smoothing. The peak surface wind speed also momentarily gusted to over $85 \mathrm{~ms}^{-1}$, but then weakened to previous levels. Surprisingly, the minimum surface pressure continued on the same trend downward it began at 45 hours and then stabilized at between 922 and $925 \mathrm{mb}$.
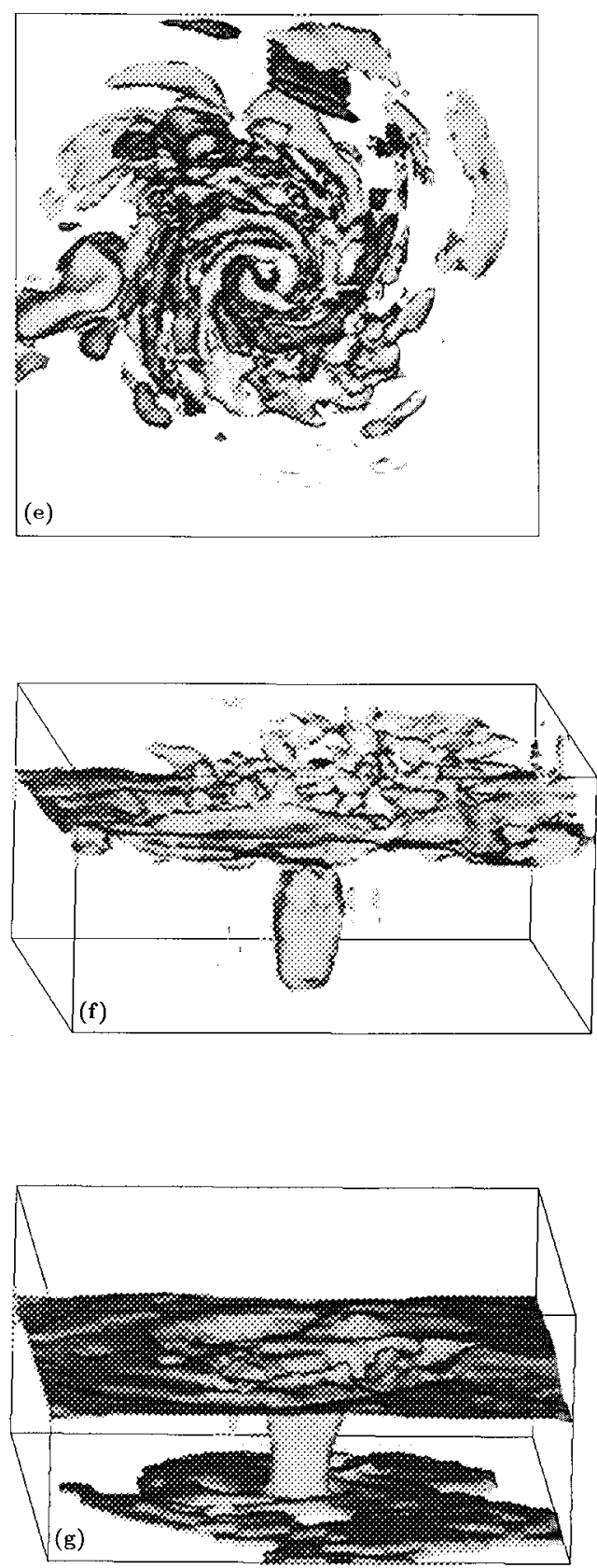

Figure 11 shows the predicted three-dimensional fields after 56 hours. Overall, they did not look substantially different than they did with the $10 \mathrm{~km}$ resolution when interpolated to the second grid. Some finer structure of the inner bands and within the TKE field was apparent.

To better depict the structure of the inner core, selected fields of the simulated eye wall within the 4th grid were displayed with full resolution. Figure 12 depicts the cloud, TKE, $w$, potential 

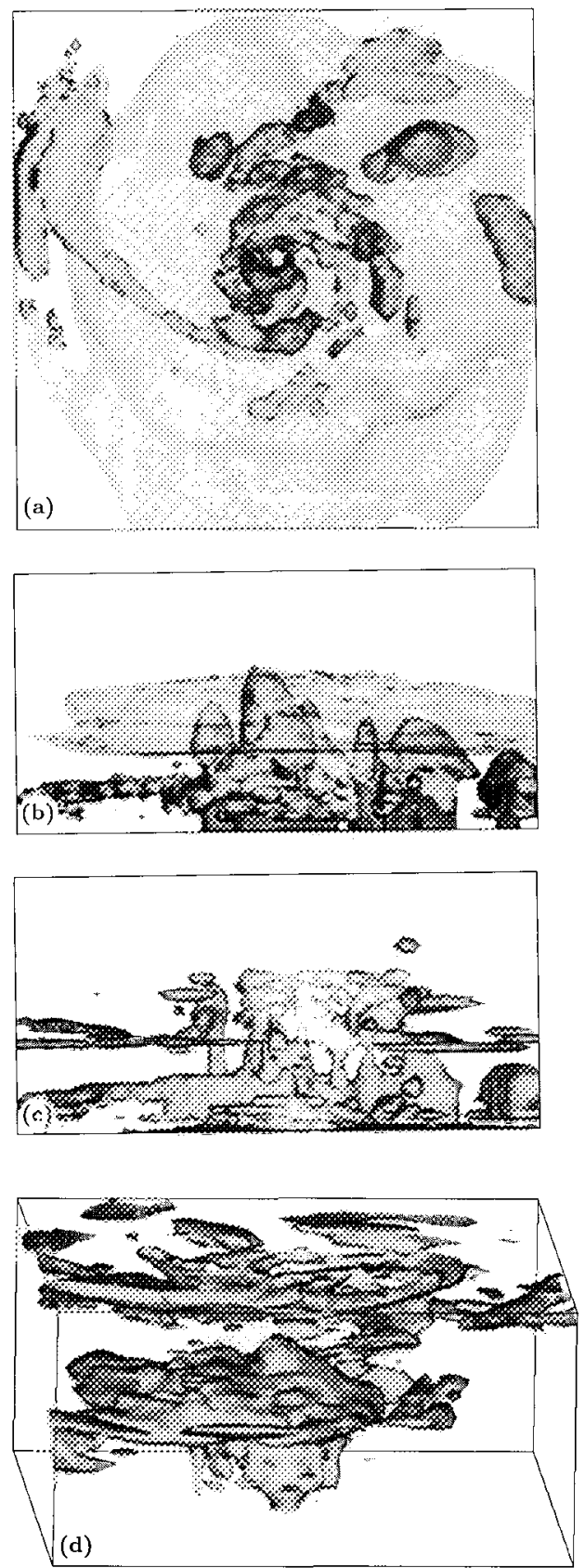

Fig. 11. Same as Fig. 4, except for $6 \mathrm{hr}$ of integration

vorticity and $\theta_{e}$ fields on the high resolution grid. Here the fine structure of the spiraling vertical motion field was readily apparent. The eye wall was constructed of several asymmetric bands of vertical motion. The cloud field was shown to almost totally lack cumulus structure and instead
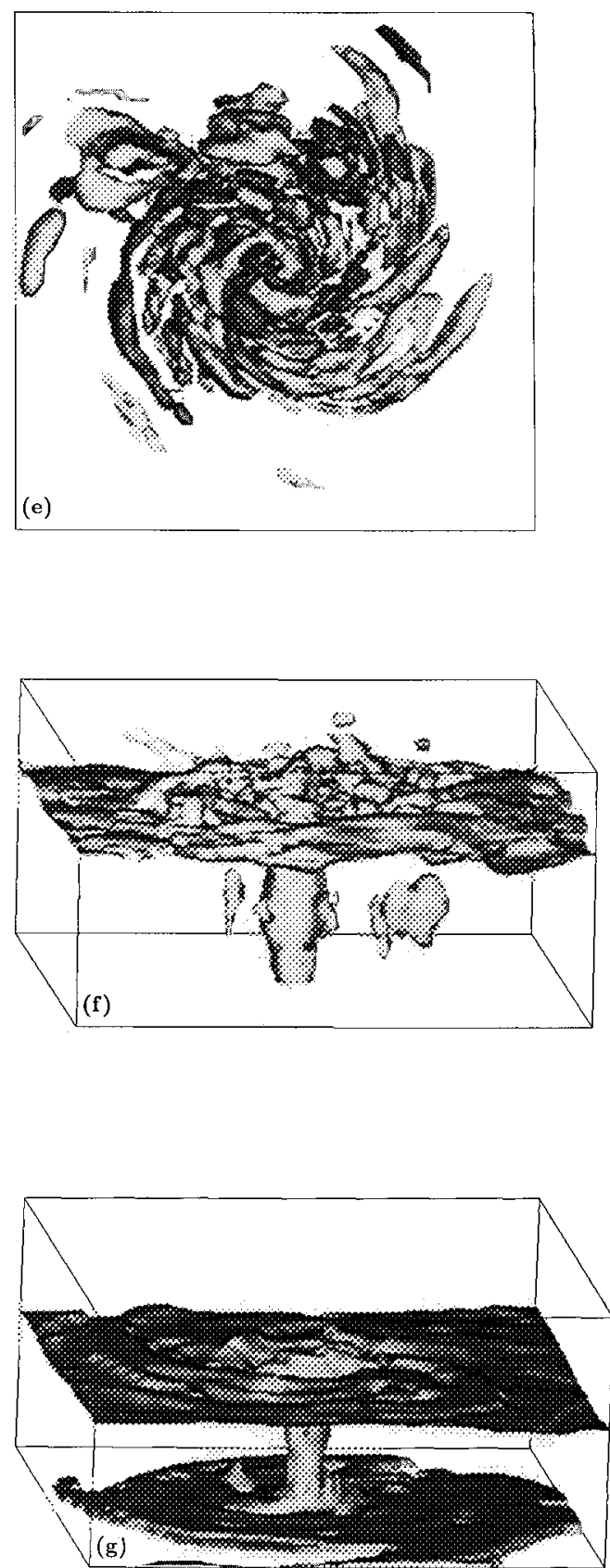

gave the appearance of a spiral shaped stratiformlike cloud. The spiral structure appeared strongly in the upper surface of the cirrus canopy, as was commonly observed by satellite.

Note also the more continuous spiraling appearance of the $\theta_{e}$ surface maximum. This was a 

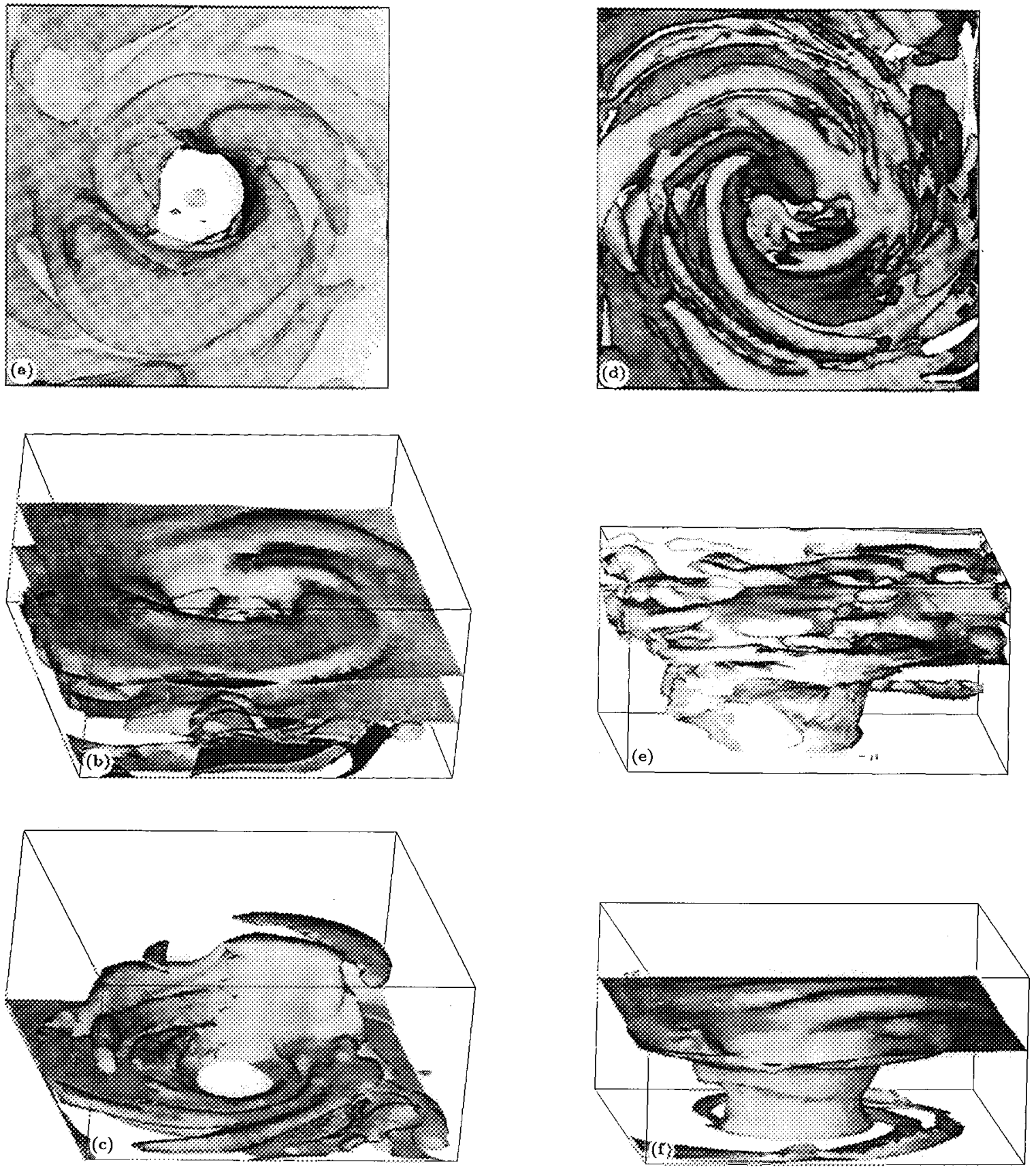

Fig. 12. The simulated three-dimensional storm structure viewed over the fourth grid (region is $198 \mathrm{~km}$ across) and at $56 \mathrm{hr}$ showing: (a) an overhead view of the $0.01 \times 10^{-6} \mathrm{gm}^{-3}$ condensate surfaces with north pointing up (the rain and graupel surfaces are darkly shaded, the cloud water and snow surfaces are lightly shaded, and the pristine crystal surface is partially transparent), (b) oblique view of the condensate surfaces described in a) from the south showing wave like texture to upper cloud surface, (c) oblique view of the $2 \mathrm{~m}^{2} \mathrm{~s}^{-2}$ TKE surface from the northwest, (d) overhead view of the $0.5 \mathrm{~ms}^{-1}$ (lightly shaded) and $-0.5 \mathrm{~ms}-1$ (darkly shaded) vertical motion surface from below with north facing up (note east is to the left here) (e) oblique view of the 0.5 PVU surface from the south, and (f) an oblique view of the $359 \mathrm{~K} \theta_{\mathfrak{e}}$ surface from the south 
result of the higher resolution depiction of the eye wall density current next to the inwardly spiraling flow.

The TKE field was confined to a considerably smaller region of the plume. This might be expected because a greater portion of the updraft was resolved explicitly and so the subgrid scale became relatively laminar, except for some local instabilities within bands.

The potential vorticity field shows considerably more structure presented on this grid. Contour cross sections through the field at low levels (not shown here) show that the potential vorticity was layered into the main center forming a spiral itself.

The inertial stability can be defined as:

$I^{2}=(\zeta+f)\left(\frac{2 V_{T}}{R}+f\right)$

where $\zeta$ is the relative vorticity, $f$ is the coriolis parameter, $R$ is the distance to the vortex center, and $V_{T}$ is the tangential wind of the vortex.

Examination of the low level inertial field (not shown) showed that the minimum of potential vorticity within the spiral was associated with a minimum of inertial stability just outside the eye wall. In fact, the inertial stability formed a spiral relative to the near spiral bands and even became negative within the split where the band formation was occurring at 56 hours. The inertial stability was maximum within the band; but still very strong within the eye itself. Hence where outward propagating bands were forming, a strong gradient of inertial stability was apparent across the region of convection.

This explains why the outer edge of the eye wall convection split forming the band. On the inside of the eye wall, the subsidence warming by the convection resulted in gradient wind adjustment and thus not weakening the eye wall. On the outside edge of the eye wall, angular momentum transported in and brought around the storm led to weakened inertial stability. This caused convergence into the eye wall to be weakened, throwing the eye wall convection into a breakdown mode. This in turn caused the surface pressure in that region of the eye wall to rise while the pressure outside the eye wall had fallen. This weakened the updraft and gave rise to an outwardly propagating inertia-gravity wave.

It is clear that a coherent relationship between the stratospheric wave and the tropospheric wave was maintained throughout the inertia-gravity wave spawning process. The extent to which resonance helped determine the frequency of convection, the optimal storm rotation rate and a host of other possibilities, will remain an intriguing topic for future research.

\section{Discussion of Scale Interactions}

The above results described several scale interaction processes occurring during the simulated tropical cyclogenesis. These processes have not been widely discussed before, primarily because previous numerical simulations have used simplified hydrostatic, axisymmetric and cumulus parameterized frameworks which poorly represent such processes. Although all of the processes described have not been proven to exist in nature, their existence in this simulation raises interesting questions and gives valuable insight into how three-dimensional tropical cyclones might explicitly interact with convection. The following is a brief summary of the major types of scale interaction noted and their impact on the lifecycle of the simulated storm.

\subsection{Simulated Mesoscale Convective Systems}

Convective plumes were simulated to preferentially separate into meso- $\beta$ scale entities in the early stages of storm development, before strong inertial balance evolved. These entities, showing both a strong gradient balanced and divergent part, appeared in the pressure field as Rossby wave-like disturbances as described by Challa and Pfeffer (1980). The divergent part of the circulation, driven by the latent heating aloft, acted to contract the vorticity field to produce the wave.

The simulated embedded MCSs underwent growth and breakdown cycles similar to those described by Tripoli and Cotton (1989a). These cycles were characterized by a period of growth where low level convergence was drawn into the system at sufficient magnitudes to fuel the system growth, while divergence or detrainment was produced at upper levels. When low level convergence was interrupted, the level of no horizontal divergence in the updraft moved upward leading to the entrainment of lower $\theta_{e}$ air and further interruption of the fuel supply. The process has been described theoretically by Raymond (1984) for the wave-CISK problem, where the growth 
process was described as an advective mode of unstable growth. Raymond found breakdown occurred when the surface divergence/convergence was upset giving rise to propagating modes of inertia-gravity waves.

The breakdown process, once initiated continues because the propagating gravity waves become the growth modes. This process was fundamentally similar to that which led to the breakdown of single cumulus plumes on the 20 minute time scale except for the processes which initiate the breakdown phase. Hence, the field of growing and collapsing MCSs continuously emitted inertia gravity waves which move about the storm, and eventually dissipate or move into the stratosphere where the model's absorbing layer removed them.

\subsection{Gravity Wave Formation Induced by Locally Weakened Inertial Stability}

Asymmetric surface deformation fields produced by convergence of flow into the developing ring of maximum winds produced a tangentially varying angular momentum field, which when advected around the vortex produced regions of weak inertial stability or inertial instability. These instabilities locally weakened the convergent flow into the eye wall by allowing the flow to respond to subsidence warming outside the eye wall. This could initiate the local breakdown of convection in the eye wall and could give rise to the formation of a propagating inertia gravity waves in the form of outward propagating bands of vertical motion, temperature and pressure. These waves, which propagated radially outward at approximately $35 \mathrm{~ms}^{-1}$, appeared to be always coupled with a stratospheric wave of similar phase speed.

The relationship between the upper and lower inertia-gravity waves was by no means just a coincidence, since the breakdown of the eyewall convection at lower levels was also tied to a collapsing cloud top. It is interesting that the growth and breakdown process seemed to take on a continuous form in the eye wall. The convection moved around the eye wall with different sections of the eye wall containing convections at different stages of the process, and thus resulting in the continuous emission of spiral gravity wave activity. At later times, the simulation seemed to prefer a tangential wave number one, with one dominate side of upward motion. There were periods however, where more continuous eye wall convective activity was apparent.

\subsection{Simulated Eye Wall During the Hurricane Stage}

As the tangential wind of the vortex increased in the hurricane stage, and the core reaches greater inertial stability, the tendency for the low level wave like MCSs within the eye wall jet were suppressed. Whereas individual air trajectories move vertically in the early phase of the storm within the individual convective systems, the grid scale air trajectories in the eye wall move in a cyclonic upward spiral during the hurricane stage, suggesting a dominance of the inertial frequency over the convective frequency within the eye wall. Since the predicted TKE magnitudes remained high in the eye wall, this motion could be interpreted to mean that individual convective plumes may have remained, although perhaps not always traversing the entire depth of the troposphere. On the other hand, MCSs, or substorm scale groupings of convective plumes were eliminated during the hurricane stage in favor of a single eye wall system.

The lowered production of gravity wave energy in a hurricane has been discussed before as a natural consequence of the geostrophic adjustment process (Schubert and Hack, 1982). The lesser production of gravity wave energy has been linked to greater efficiency of growth and hence an acceleration of the growth rate of tropical cyclones as they approach maturity. In this simulation, this process was seen explicitly as a changing form of the mesoscale convective entity from cyclic convective entities to a quasi-steady eye wall circulation.

\subsection{Simulated Eye During the Hurricane Stage}

Another consequence of the inertial stabilization of the eye wall convection was the formation of a true "eye". In the early depression and tropical storm stages, an intermittent clearing in the storm center resulted by the emphasis of convection along the annulus of high winds where the surface fluxes of $\theta_{e}$ were highest. This appearance would be less likely if a mean wind was considered, which would somewhat negate that effect. The early eye-like structure exhibited only intermittent subsidence within and was too large in diameter. 
As the storm reached hurricane stage, however, and upward motion became more axisymmetric along the eye wall, mean subsidence developed to the surface producing an eye $20-30 \mathrm{~km}$ in diameter. This resulted because the inertial frequency of the storm increased to exceed that of the formation of divergent wind in response to the convective heating. As a result, the central warming no longer forced nor fueled convective breakdown in the eye wall and became long-lived.

\subsection{Microphysics Feedbacks}

It was found that the convective regions produced surface $\theta_{e}$ minima, primarily as a result of extensive melting in the $3-5 \mathrm{~km}$ AGL layer. These surface $\theta_{e}$ minima outside the eye wall showed long-term persistence and, once formed, tended to be associated with more long term convection.

The $\theta_{e}$ minima, became entrained into the inward spiral of inflow and seemed to be focal points for the initiation of new convection by spirally banded banded gravity waves passing overhead.

The ice anvil also appeared to have a substantial impact on storm structure and growth. It is expected that the radiative feedback was of importance because of its affect on increasing upper tropospheric stability during the day and decreasing it during the night. This may have helped to duct tropospheric gravity waves beneath the unstable anvil top, enabling spiral cloud band formation at greater distances from the core.

The other major impact hypothesized was the anvil's impact on low level stability as a result of the virga falling into the $\theta_{e}$ minimum. This would have tended to weaken tropospheric stability to the point that outwardly propagating inertiagravity waves could more easily initiate clouds or unstable convection.

The present results combined with earlier studies such as Kanak (1990), Kanak and Tripoli (1990) and other unreported preliminary experiments suggest, that in the absence of a high ice water content in the cirrus anvil, the diurnal radiative effect of the anvil dominates and the existence of outlying convective activity took on a diurnal fluctuation in intensity. When essentially no anvil was present, outlying convection became virtually nonexistent. Hence radiative transfer combined with microphysical processes seemed to have a dramatic influence on storm intensity, structure, growth rate and nature of internal scale interaction.

\section{Conclusions}

This paper described nonhydrostatic and threedimensional scale interaction simulations of a tropical cyclone. The numerical model successfully simulated tropical cyclogenesis using a 1.5 level turbulence closure to represent cumulus activity on a meso- $\beta$ scale nested grid system. Although the simulation was not of a real storm situation, the storm genesis from a prescribed initial vortex appeared to be realistic. Important features of simulated scale interaction were demonstrated, some of which have been implied in the literature previously and some of which are apparently new. The major conclusions are as follows:

- The scale interaction within the simulated tropical cyclone occurred in two major phases summarized as follows:

(1) The "tropical depression" stage made up the first phase of scale interaction, and was characterized by the proliferation of meso$\beta$ scale growing and decaying convectively forced disturbances which spawned deep propagating inertia-gravity waves. Waves were spawned in both the stratosphere and troposphere. The amplitude of the pressure ocscillation beneath the MCSs exceeded the central pressure of the storm.

(2) The "tropical storm" stage marked the period where the storm's central pressure exceeded the amplitude of the fluctuation in the surrounding convection. This enabled a continuous warm core to grow and maintain mean subsidence for increasingly long periods. Convective entities within the eye wall became longer lived as a result of an increasing portion of the induced warming being captured within the inertially stable eye wall without loss of surface convergence.

(3) The "hurricane" stage was characterized by inertially balanced eye-wall convection which maintained continuous eye subsidence which allowed the subsidence to work it way to the surface. At that time the cyclone pressure became nearly axisymmetric inernally although important asymmetries existed outside the eye wall due to inertia-gravity 
wave activity. Convection in the eye wall became less cellular with updrafts taking on the scale and geometry of the eye wall itself. The eye wall at times seemed to behave as a single convective cell.

- Simulation with a meso- $\gamma$ resolving grid of $3.3 \mathrm{~km}$ spacing within the eye wall had a surprisingly small impact on the storm evolution, although much smoother and more coherent looking features were simulated. The results suggested that a $10 \mathrm{~km}$ horizontal resolution was likely sufficient to approximate mature tropical cyclone dynamics.

These simulations crudely begin to unfold the explicit scale interaction processes which exist, but offer the reader little confidence that the answer has been found. Instead, they instill a sense of uneasiness that there are important essential processes within convective weather systems that have never been addressed theoretically or within predictive model frameworks. As research numerical models become capable of better representing these process by simulating a greater range of wave numbers using larger grids, a better understanding of the processes will be achieved, new questions will be posed and requirements for accurate prediction will be made.

\section{Acknowledgements}

The author would like to thank Kathy Kanak, who helped develop the tropical cyclone model and analysis system and ran the first high resolution three-dimensional experiments. Thanks also to Pete Pokrandt for useful comments and his help with model development. Thanks to Mr. Brain Paul for his considerable help in customizing visualization software for this paper. Jill Bushner and Bonnie Tripoli helped editing the manuscript and preparing the figures. The numerical computations were performed, on the NCAR (National Center for Atmospheric Research) CRAYs and on the University of Wisconsin-Madison Space Sciences and Engineering Center Stellar GS-2000 super graphics work station. NCAR is partially supported by the National Science Foundation. This work was supported under NSF Grants ATM-88-05460 and ATM-91-01434 and NASA grant NAG8828 for the visualization.

\section{Appendix A}

\section{Level 1.5 Closure}

The level 1.5 closure scheme is based on the scheme presented by Redelsperger and Sommeria (1981) (hereafter referred to as RS) with some modification. For a complete derivation, the reader is referred to that paper. Here, the modifications to the RS scheme are presented and the final form of the implementation of the scheme is given.

The closure simply involves an alternate procedure for computing the mechanical $\left(K_{M}\right)$ and thermal $\left(K_{H}\right)$ eddy mixing coefficients used in the second order diffusion term. In the case of the hurricane, the scheme is only applied to the calculation of vertical eddy mixing coefficient. The TKE is defined as:

$e=0.5 u_{i}^{\prime 2}$,

where $u_{i}$ is the three dimensional velocity tensor and the prime refers to the turbulent deviation from the resolved scale. Retaining only the largest order terms defined in RS, the vertical mechanical mixing coefficient is defined as:

$K_{m}=C_{m} l_{z} e^{1 / 2} \frac{1-\max \left(0, C_{1} R_{i}\right)}{1+\max \left(0, C_{1} R_{i}\right)}$

where $l_{z}$ is the vertical grid spacing, $C_{m}=\frac{1}{15}$, and $R_{i}$ is the Richardson number, given by:

$R_{i}=\frac{N^{2}}{D_{i j}^{2}}$,

where $N$ is the Brunt-Vaisala frequency and $D_{i j}$ is the deformation tensor. The constant $C_{1}$ is defined to be:

$C_{1}=\frac{2}{3 C C_{\theta}}$,

where $C=4.0$ and $C_{0}=1.2$. The thermal eddy mixing coefficient is related to the mechanical mixing coefficient by the formula:

$\frac{K_{H}}{K_{M}}=\frac{5}{2} \frac{C_{M}}{C_{H}}$

where $C_{H}=4$.

The tendency equation for TKE is given by:

$$
\begin{aligned}
\frac{d e}{d t}= & -C_{M} l_{z} e^{1 / 2}\left(D_{i j}^{2}-\frac{K_{H}}{K_{M}} N^{2}\right) \frac{1-\max \left(0, C_{1} R_{i}\right)}{1+\max \left(0, C_{1} R_{i}\right)} \\
& -C_{\varepsilon} l_{z}{ }^{-1} e^{2 / 3}-e \frac{2}{3} \frac{\partial u_{i}}{\partial x_{i}},
\end{aligned}
$$

where $u_{i}$ is a velocity tensor, $x_{i}$ is the cartesian distance, and $C_{\varepsilon}$ is the dissipation coefficient which is set to $C_{\varepsilon}=0.7$ except near the surface where it is ramped up to $C_{\varepsilon}=2.7$.

The buoyancy production (term involving $N^{2}$ in A6) is the primary term driving the development of deep subgrid scale moist convective overturning. The vertical thermal buoyancy driven acceleration of velocity can be defined as:

$\left(\frac{d u_{3}}{d x_{3}}\right)_{\text {buoyancy }}=g \frac{\theta_{v i l}^{\prime}}{\theta_{0}}$,

where $\theta_{0}$ is a reference state potential temperature and $\theta_{\text {vil }}$ is a mixed phase virtual potential temperature defined as:

$\theta_{v i l}^{\prime}=\theta \frac{1+0.61 q_{v}}{1.0+q_{l}+q_{i}}-\theta_{0}$

where $\theta$ is the potential temperature, $q_{v}, q_{l}$, and $q_{i}$ are the 
specific humidities of vapor, liquid and ice in the system. This form of virtual potential temperature is useful because it takes into account both the effects of vapor and water loading in addition to that of temperature in creating buoyancy.

Following RS, the buoyancy production term is related to the vertical heat flux by:

$\frac{g}{\theta_{0}} \overline{u_{3}^{\prime} \theta_{v i l}^{\prime}}=-K_{H} \frac{g}{\theta_{0}}\left(\frac{\partial \theta_{v i l}^{\prime}}{\partial x_{3}}-\frac{L_{m}}{c_{p} T} \frac{d q_{v}}{d x_{3}}\right)=-K_{H} N^{2}$,

where $c_{p}$ is the specific heat at constant pressure, $T$ is the temperature and $L_{m}$ is the latent heat of phase change defined as:

$L_{m}=w_{l} L_{v l}+\left(1+w_{l}\right) L_{v i}$

where $L_{v i}$ and $L_{v i}$ are the latent heats of evaporation and sublimation respectively and $w_{l}$ is a weighting function used to represent the change from liquid to ice in the temperature range of $253-273 \mathrm{~K}$ given by:

$w_{l}=\min \left(1, \max \left(0, \frac{T-273.16}{20.0}\right)\right)$.

The latent heating term is to take into account the effects of heat released or absorbed by phase changes associated with either saturated ascent or melting and evaporation on unsaturated decent. The vapor change is defined as:

$d q_{v}= \begin{cases}d q_{m} & \text { for } q_{v}>q_{s} \\ E_{p}-\min \left(d q_{m}+\left(q_{s}-q_{v}\right), q_{l}+q_{i}\right) & \text { for } q_{v} \leqslant q_{s}\end{cases}$

where $q_{s}$ is the phase weighted saturation mixing ratio, $E_{p}$ is an assumed precipitation vaporization efficiency, taken here to be $E_{p}=0.5$, and $q_{m}$ is the change in vapor mixing ratio due to phase change over one grid volume which can be estimated (Cotton and Tripoli, 1978) as:

$d q_{m}=\frac{g \Delta z q_{v}}{R T} \frac{\frac{\varepsilon L_{m}}{c_{p} T}-1}{\frac{\varepsilon L_{m} q_{v}}{R c_{p} T^{2}}-1}$

where $R$ is the gas constant and $\varepsilon=0.622$.

The Brunt-Vaisala frequency implied by Eq. A9, is also used to define the Richardson number. This from of the Brunt-Vaisala frequency has been found to effectively force deep mixing plumes, which represent cumulus reasonably well, when the circulation is partially resolved by the grid.

\section{Appendix B}

\section{Rankine Vortex Initialization}

The initial wind circulation is built from a specified vorticity field by solving the Poisson equation for stream function. The vorticity field is given by:

$\zeta=\left\{\begin{array}{ll}w_{\xi}\left(x_{3}\right)\left(\zeta_{1}-\zeta_{2}\right) & \text { for } R<R_{2} \\ 0 & \text { for } R \geqslant R_{2}\end{array}\right.$,

where $\zeta, \zeta_{1}$, and $\zeta_{2}$ are relative vorticity values for the vortex and two components comprising the vortex respectively and $R$ and $R_{2}=800 \mathrm{~km}$ are the great circle distances to the vortex center for an arbitrary location and the location and the outer limits of the circulation respectively. The vortex strength is varied with height by the relationship:

$w_{\xi}\left(x_{3}\right)=\left\{\begin{array}{ll}1 & \text { for } x_{3}<z_{1} \\ e^{\left(-x_{3}-z_{1} / z_{2}-z_{1}\right)} & \text { for } x_{3} \geqslant z_{1}\end{array}\right.$,

where $z_{1}=10 \mathrm{~km}$ and $z_{2}=13 \mathrm{~km}$. The inner vorticity component is defined:

$\zeta_{1}=\zeta_{\max } e^{\operatorname{In}(0.1)\left(R / R_{1}\right)^{4}}$,

where $R_{1}=400 \mathrm{~km}$ is the radius by which the inner vorticity is reduced by one order of magnitude, and $\zeta_{\max }=2.0 \times 10^{-4}$ is the center vorticity value.

The outer vorticity field is added to force the average vorticity added by the perturbation to zero and is given by:

$\zeta_{2}=\sin \left(\pi \frac{R}{R_{2}}\right) \frac{\left[\zeta_{1}\right]}{\left[\sin \left(\pi \frac{R}{R_{2}}\right)\right]}$

where the horizontal averaging operator is defined for some arbitrary variable $\Psi$ to be:

$[\Psi]=\frac{\int_{A} \Psi d A}{\int_{A} d A}$,

where $A$ is the area over which the average is taken, which in this case, is the area within $R_{2}$.

\section{References}

Arakawa, A., Schubert, W. H., 1974: Interaction of a cumulus ensemble with the large scale environment. Part I. J. Atmos. Sci., 31, 674-701.

Challa, M., Pfeffer, R. L., 1980: Effects of eddy fluxes of angular momentum on model hurricane development. $J$. Atmos. Sci., 37, 1603-1618.

Charney, J., Eliassen, A., 1964: On the growth of the hurricane depression. J. Atmos. Sci., 21, 68-75.

Chen, C., Cotton, W. R., 1983: A one-dimensional simulation of the stratocumuluscapped mixed layer. Bound.-Layer Meteor., 25, 289-321.

Clark, T. L., 1977: A small scale dynamic model using a terrain-following coordinate transformation. J. Comp. Phys., 24, 186-215.

Clark, T. L., Farley, R. D., 1984: Severe downslope windstorm calculations in two and three spatial dimensions using anelastic interactive grid nesting: A possible mechanism for gustiness. J. Atmos. Sci., 41, 329-350.

Cotton, W. R., Tripoli, G. J., 1978: Cumulus convection in shear flow - three-dimensional numerical experiments. J. Atmos. Sci., 35, 1503-1521.

Cotton, W. R., Tripoli, G. J., Rauber, R. M., Mulvihill, E. A., 1986: Numerical simulation of the effects of varying ice crystal nucleation rates and aggregation on the processes on orographic snowfall. J. Climate. Appl. Meteor., 25, $1658-1680$. 
Delsol, F., Miyakoda, K., Clarke, R. H., 1971: Parameterized processes in the surface boundary layer of an atmospheric circulation model. Quart.J. Roy. Meteor. Soc., 97, 181-208.

Durran, D. R., 1981: The effects of moisture on mountain lee waves. Technical Report PhD Thesis NTIS PB82156621, Massachusetts Institute of Technology.

Fritsch, J. M., Chappel, C. F., 1980: Numerical simulation of convectively driven mesoscale pressure systems. Part I. Convective parameterization. J. Atmos. Sci., 37, 1722-1733.

Holland, G. J., 1983: Angular momentum transports in tropical cyclones. Quart. J. Roy. Meteor. Soc., 109, 187-209.

Kanak, K. M., 1990: Three-Dimensional Nonhydrostatic Numerical Simulation of a Developing Tropical Cyclone. Master's thesis, University of Wisconsin-Madison.

Kanak, K. M., Tripoli, G. J., 1990: Three-dimensional, nonhydrostatic simulation of scale interaction within a tropical cyclone. In: Preprints, Fourth Conference on Mesoscale Processes, Boulder, Colorado, pp. $28-29$. Boston, MA: American Meteorological Society.

Klemp, J. B., Wilhelmson, R. B., 1978: The simulation of three-dimensional convective storm dynamics. J. Atmos. Sci., 35, 1070-1096.

Kreitzberg, C. W., Perky, D. J., 1976: Release of potential instability: Part I. A sequential plume model within a hydrostatic primitive equation model. J. Atmos. Sci., 33, 456-475.

Kurihara, Y., 1976: On the development of spiral bands in tropical cyclones. J. Atmos. Sci., 33, 940-958.

Lindzen, R. S., 1974: Wave-CISK in the tropics. J. Atmos. Sci., 31, 156-179.

Louis, J. F., 1979: A parameteric model of vertical eddy fluxes in the atmosphere. Bound.-Layer Meteor., 17, 187-202.

Ooyama, K., 1964: A dynamical model for the study of tropical cyclone development. Geofis. Intern., 4, 187-198.

Raymond, D. J., 1975: A model for predicting the movement of continuously propagating convective storms. J. Atmos. Sci., 32, 1308-1317.

Raymond, D. J., 1976: Wave-CISK and convective mesosystems. J. Atmos. Sci., 33, 2392-2398.

Raymond, D. J., 1984: A Wave-CISK model of squall lines. J. Atmos. Sci., 41, 1946-1948.

Redelsperger, J. L., Sommeria, G., 1981: Methode de representation de la turbulence d'echelle inferieure a la maille pour un modele tri-dimensional de convection nuageuse. Bound.-Layer Meteor., 21, 509-529.

Redelsperger, J. L., Sommeria, G., 1982: Methode de representation de la turbulence associe aux precipitations dans unmodele tri-dimensional de convection nuageuse. Bound.Layer Meteor, 24, 231-252.

Rosenthal, S. L., 1978: Numerical simulation of a tropical cyclone development with latent heat release by the resolvable scales I: Model description and preliminary results. J. Atmos. Sci., 35, 258-271.

Schubert, W. H., Hack, J. J., 1982: Inertial stability and tropical cyclone development. J. Atmos. Sci., 39, 1687-1697.

Stull, R. B., 1989: An Introduction to Boundary Layer Meteorology. Kluger Academic press.

Tremback, C. J., 1990: Numerical Simulation of a Convective Comple: Model Development and Numerical Results. $\mathrm{PhD}$ thesis, Colorado State University, Fort Collins, CO 80523.

Tripoli, G. J., 1992: A nonhydrostatic numerical model designed to simulate scale interaction. Mon. Wea. Rev., 120, (in press).

Tripoli, G. J., Cotton, W. R., 1982: The Colorado State University three-dimensional model - 1982. Part I: General theoretical framework and sensitivity experiments. J. Rech. Atmos., 16, 185-220.

Tripoli, G. J., Cotton, W. R., 1989: Numerical study of an observed orogenic mesoscale convective system. Part 1: Simulated genesis and comparison with observations. Mon. Wea. Rev., 117, 273-304.

Willoughby, H. E,, 1977: Inertia-buoyancy waves in hurricanes. J. Atmos. Sci., 34, 1028-1039.

Willoughby, H. E., 1978: A possible mechanism for the formation of hurricane rainbands. J. Atmos. Sci., 35, $838-848$.

Willoughby, H. E., 1979: Excitation of spiral bands in hurricanes by the interaction between the symmetric mean vortex and a shearing environmental steering current. J. Atmos. Sci., 36, 1226-1235.

Author's address: G. J. Tripoli, University of WisconsinMadison, Department of Meteorology, Madison, Wisconsin 53706, U.S.A.

\footnotetext{
Verleger: Springer-Verlag KG, Sachsenplatz 4-6, A-1201 Wien. - Herausgeber: Pro:. Dr. Elmar R. Reiter, Wels Research Corporation, Boulder, CO 80301, U. S. A. Redaktion: Türkenschanzstraße 18, A-1180 Wien. - Satz und Umbruch: Thomson Press (India) Ltd., New Delhi. Reproduktion und Offsetdruck: Druckerei G. Grasl, A-2540 Bad Vöslau. - Verlagsort: Wien. - Herstellungsort: Bad Vöslau. - Printed in Austria.

Offenlegung gem. $\S 25$ Abs. 1 bis 3 Mediengesetz

Offenlegung gem. $\$ 25$ Abs. 1 bis 3 Mediengesetz
Unternehmensgegenstand: Verlag von wissenschaftlichen Büchern und Zeitschriften.

An der Springer-Verlag KG ist beteiligt: Dr. Konrad F. Springer, Sachsenplatz 4-6, A-1201 Wien, als Kommanditist zu 52,38\%. Geschäftsführer: Dr. Konrad F. Springer, Prof. Dr. Dietrich Götze, Ing. Wolfram F. Joos, Dipl.-Kfm. Claus Michaletz und Rudolf Siegle, alle Sachsenplatz 4-6, A-1201 Wien.
} 